\title{
Computational Personality Recognition in Social Media
}

\author{
Golnoosh Farnadi • Geetha Sitaraman • \\ Shanu Sushmita - Fabio Celli . Michal \\ Kosinski · David Stillwell • Sergio \\ Davalos · Marie-Francine Moens · Martine \\ De Cock
}

Received: date / Accepted: date

\begin{abstract}
A variety of approaches have been recently proposed to automatically infer users' personality from their user generated content in social media. Approaches differ in terms of the machine learning algorithms and the feature sets used, type of utilized footprint, and the social media environment used to collect the data. In this paper, we perform a comparative analysis of state-of-the-art computational personality recognition methods on a varied set of social media ground truth data from Facebook, Twitter and YouTube. We answer three questions: (1) Should personality prediction be treated as a multi-label prediction task (i.e., all personality traits of a given user are predicted at once), or should each trait be identified separately? (2) Which predictive features work well across different online environments? and (3) What is the decay in accuracy when porting models trained in one social media environment to another?
\end{abstract}

Keywords Big Five personality · Social media · User generated content • Multivariate regression · Feature analysis

\section{Introduction}

Research in psychology has suggested that behavior and preferences of individuals can be explained to a great extent by underlying psychological constructs: personality traits [42]. Knowledge of an individual's personality allows us to make predictions about preferences across contexts and environments, and to enhance recommendation systems [33. Personality can affect the decision making process and has been shown to affect preferences for websites [31, products, brands and services [32], and for content such as movies, TV shows, and books [9].

This paper or a similar version is not currently under review by a journal or conference, nor will it be submitted to such within the next three months. This paper is void of plagiarism or self-plagiarism as defined in Section 1 of ACM's Policy and Procedures on Plagiarism ( http://www.acm.org/publications/panel/policies/plagiarism_policy). 
The most widely accepted model of personality, Big Five or Five Factor Model, embraces five traits [12]: Openness, Conscientiousness, Extroversion, Agreeableness, and Emotional Stability (often conversely referred to as Neuroticism). Further explanations of each trait are summarized in Table 1.

Table 1 Overview of the Big Five Personality Model.

\begin{tabular}{|c|c|}
\hline Trait & Description \\
\hline Openness & $\begin{array}{l}\text { Openness is related to imagination, creativity, curiosity, tol- } \\
\text { erance, political liberalism, and appreciation for culture. } \\
\text { People scoring high on Openness like change, appreciate new } \\
\text { and unusual ideas, and have a good sense of aesthetics. }\end{array}$ \\
\hline Conscientiousness & $\begin{array}{l}\text { Conscientiousness measures preference for an organized ap- } \\
\text { proach to life in contrast to a spontaneous one. People } \\
\text { scoring high on Conscientiousness are more likely to be } \\
\text { well organized, reliable, and consistent. They enjoy plan- } \\
\text { ning, seek achievements, and pursue long-term goals. Non- } \\
\text { conscientious individuals are generally more easy-going, } \\
\text { spontaneous, and creative. They tend to be more tolerant } \\
\text { and less bound by rules and plans. }\end{array}$ \\
\hline Extroversion & $\begin{array}{l}\text { Extroversion measures a tendency to seek stimulation in the } \\
\text { external world, the company of others, and to express pos- } \\
\text { itive emotions. People scoring high on Extroversion tend to } \\
\text { be more outgoing, friendly, and socially active. They are usu- } \\
\text { ally energetic and talkative; they do not mind being at the } \\
\text { center of attention, and make new friends more easily. In- } \\
\text { troverts are more likely to be solitary or reserved and seek } \\
\text { environments characterized by lower levels of external stim- } \\
\text { ulation. }\end{array}$ \\
\hline Agreeableness & $\begin{array}{l}\text { Agreeableness relates to a focus on maintaining positive so- } \\
\text { cial relations, being friendly, compassionate, and coopera- } \\
\text { tive. People scoring high on Agreeableness tend to trust oth- } \\
\text { ers and adapt to their needs. Disagreeable people are more } \\
\text { focused on themselves, less likely to compromise, and may } \\
\text { be less gullible. They also tend to be less bound by social } \\
\text { expectations and conventions, and more assertive. }\end{array}$ \\
\hline Emotional Stability & $\begin{array}{l}\text { Emotional Stability, reversely referred to as Neuroticism, } \\
\text { measures the tendency to experience mood swings and emo- } \\
\text { tions such as guilt, anger, anxiety, and depression. People } \\
\text { scoring low on Emotional Stability (high Neuroticism) are } \\
\text { more likely to experience stress and nervousness, while peo- } \\
\text { ple scoring high on Emotional Stability (low Neuroticism) } \\
\text { tend to be calmer and self-confident. }\end{array}$ \\
\hline
\end{tabular}

A traditional approach to measure personality requires participants to answer a series of questions (typically, from 20 to 360) evaluating their behavior and preferences (e.g. [28, 12]). This approach is time-consuming and impractical, especially in the context of on-line services. On-line users might be unwilling to spend a considerable amount of time filling-in a questionnaire, in order to personalize their search results or product recommendations.

However, it has been recently shown that the digital footprint of users can be used to automatically infer their personality. For example, 32 and [55] showed that automated personality judgments based on Facebook Likes are more accurate than those made by users' friends or even their spouses. Also, 43. showed that similar predictions can be based on language used in social media. A variety of other 
approaches have been proposed using different prediction mechanisms, feature spaces, and focusing on different on-line environments [1, 15, 46].

\section{Aims of the Study}

In this study, we perform a comparative analysis of state-of-the-art computational personality recognition methods on a varied set of social media benchmark datasets collected on Facebook, Twitter and YouTube. Our aim is to address the three following questions.

(1) Should personality prediction be treated as a multi-label prediction task (i.e., all personality traits of a given user are predicted at once), or should each trait be identified separately?

Given the user generated content of each user, the aim is to obtain a set of five estimates (real numbers) representing the Big Five dimensions. We treat this problem as a regression problem by exploring different univariate and multivariate regression techniques. Recently, research has been done on the use of multivariate regression for personality prediction on Facebook [27] and YouTube [15.

In this study, we compare multivariate regression techniques, e.g., multi-target stacking, ensemble of regressor chains, and multi-objective random forests [54, with univariate approaches such as support vector machines and decision trees, as well as with an average baseline algorithm. The average baseline predicts for each data point the mean value across the training data (e.g. if the average openness score of all users in the training data is 2.5 , then it predicts that value as the openness score for all users in the test data).

(2) Which predictive features work well across different on-line environments?

We extract a wide variety of linguistic and emotional features from Facebook status updates, tweets and transcripts of vlogs (i.e., video blogs). The underlying rationale for including linguistic and emotional features is that people with different personality traits will express themselves differently and, hence, will use different words (phrases) and emotions (anger, joy).

We assess the strength of the relationship between different predictive features and the personality traits by determining their correlations. We compare the correlation results across different datasets. Finding correlations of text-based features with personality traits has been previously studied (e.g., [43, 51,46]). However, to the best of our knowledge, there is no work that compares the results over different benchmark datasets. We select features according to their relationship with personality scores. Motivated by previous research, and the observed correlation between features and personality scores, we include them in our regression models. Our aim is to determine which relationships between features and personality traits are common across various social media platforms.

(3) What is the decay in accuracy when porting models trained in one social media environment to another?

Personality predictions are challenging; unlike demographic data, ground truth (i.e., questionnaire scores) is relatively scarce and measured with a considerable error. Farnadi et al. 16] suggested cross-learning, or developing personality prediction models using a variety of digital environments. The advantage of cross-learning is that training examples from different social media platforms can be combined 
to increase the accuracy on other test data. Such models could also be applied to environments where training data representative for the deployment domain is not available. In this study, we explore the possibilities of cross-learning for personality prediction by using benchmark datasets from three different environments (i.e., Facebook, YouTube and Twitter).

\section{Related Work}

In this section we present background material that supports this study. In particular, state-of-the-art efforts related to users' personality predictions, their associated preferences and behavior are provided. In addition, we also describe related work that uses different social media data like Facebook, Twitter and YouTube for the purpose of personality prediction tasks and their analyses.

Personality Prediction, Preference and Behavior: Knowledge about an individuals' personality can allow us to make predictions about preferences across contexts and environments, and enhance recommendation systems [25, 41]. Previous work in the field of psychology and human computer interaction (HCI) has highlighted the importance of identifying users' personality traits and their preferences. This can help in building adaptive and personalized systems in order to provide rich and improved user experiences 40 . For instance, in order to understand the online profile creation process, Counts and Stecher [13] conducted a study, and found that free-form profile attributes allow best desired self presentations, and only specific attributes were needed for sufficient self presentation. In a separate study by Lee and Nass 35, interaction effects between user factors, and media factors on feelings of social presence were investigated. It was found that matching synthesized voice personality to the user personality positively affects users' (especially extrovert users') feelings. Such findings can be critical in the design of virtual reality systems and human computer interfaces. In a study by Saati et al. [50] it was found that extroverts tended to interact faster with the user interface than introverts. The study also suggests that personality data could help designers to select appropriate skin colours for the user interface.

Identifying users' personality is not only useful for commercial purposes, but it can also help in understanding the mental health and high risk factors of online users. For instance, [18] examined the relationship of Social Networking Sites (SNS) and their problematic usage with regard to personality characteristics and depressive symptomatology. The results of this study indicate that problematic SNS usage is significantly and positively related to depression and Neuroticism, while being negatively associated with Agreeableness.

Social Media and Personality: Social media websites provide a unique opportunity for personalized services to capture various aspects of user behavior. Besides users' structured information contained in their profiles, e.g., demographics, users produce large amounts of data about themselves in a variety of ways including textual (e.g., status updates, blog posts, comments) or audiovisual content (e.g., uploaded photos and videos). Many latent variables such as personalities, emotions and moods - which, typically, are not explicitly given by users - can be extracted from user generated content (see e.g. 4, 16, 20]). Research into automatic personality prediction using social media data is a very nascent area which 
is gaining increased research attention due to its potential in many computational applications.

Next, we discuss the relevant background material on how different social media data like Facebook, Twitter and YouTube have been used individually by researchers for the purpose of personality prediction tasks and analyses. Note that, in this study, our aim is to perform a comparative analysis of state-of-the-art computational personality recognition methods on a varied set of social media ground truth data from Facebook, Twitter and YouTube.

Facebook Dataset and Personality: In recent years there have been several dedicated research efforts that utilized Facebook data collected as part of the myPersonality project (e.g., $23,3,47,16,9,14$ ). The details of this dataset are described in Section 4.1. In a study by Hagger-Johnson et al. [23, extracted data from the interests and activities sections of Facebook profiles were used to compare general personality and Sensational Interests Questionnaire (SIQ) scores. Sensational interests are interests that are unusually violent such as weapons, martial arts, etc.

Bachrach et al. used the myPersonality Facebook dataset to investigate how users' activity on Facebook relates to their personality. One of the findings was that Neuroticism has a generally significant negative correlation with the number of friends. The results also showed some evidence that Agreeableness is positively correlated with the number of tags. In a study by Farnadi et al. [16] the relation between emotions expressed in Facebook status updates and the users' age, gender and personality were investigated. Several interesting observations were made through this study. For instance, it was found that extrovert and open users are more emotional in their status posts than neurotic users. Another example of research that utilized the myPersonality Facebook data is the study by Cantador et al. 9]. The authors used the Facebook dataset to investigate the relations between personality types and user preferences in multiple entertainment domains, namely movies, TV shows, music, and books. In this paper, we also use the Facebook dataset from the myPersonality project.

Twitter Dataset and Personality: User generated content on Twitter (e.g., tweets) also provides a valuable source of information for inferring users' personality traits. One of the Twitter datasets often used in the literature is collected through the myPersonality project. Among thousands of participants involved in the myPersonality project, only a few hundreds of users posted links to their Twitter accounts, which forms the content of this dataset. This dataset has been used for the task of automatically predicting the personalities of the users, as well as for user behavior analyses [46,26, 19]. For instance, Quercia et al. [46] found that extroverts and emotionally stable people are popular as well as influential users on Twitter. It was also observed that popular users are imaginative, while influential people on Twitter are more organized. Golbeck et al. 2011 [19] used profile information from the dataset as features when training machine learning algorithms to predict scores on each of the five personality traits that were predicted within $11 \%-18 \%$ of their actual value. On the other hand, Hughes et al. 2012 [26] collected a different dataset from Twitter through an advertisement posted on both Twitter and Facebook. The findings of their study revealed a differential relationship between behaviors on Facebook and Twitter. It was also found that there were personality differences between those who have a preference for Facebook or Twitter, suggesting that different people use the same sites for different purposes. 
The Twitter dataset that we collected for this study (described in Section 4.3) is a new dataset, hence no previous works are based on it.

YouTube Dataset and Personality: Analysis of video content appears to be one of the least studied problems in the domain of computational personality recognition [6]. A recently collected and annotated YouTube dataset (see Section 4.2 for a detailed description) has sparked interest in personality recognition of vloggers (i.e., video bloggers). The task at hand is different from the work on computational personality recognition in the other social media platforms described above, in the sense that the ground truth data does not come from the vloggers themselves, but from other users watching the videos made by the vloggers. In other words, the task being addressed is not recognition of the true personality traits of vloggers, but predicting how the personality of vloggers is perceived by their viewers.

For instance, Aran and Gatica-Perez [2] used this data for a comparison between the personality traits extracted from YouTube and in face-to-face meetings. In another study [7, the vlog dataset was used to build personality models trained on the vlogs, and then applied to classify the EAR audio corpus. Their results suggest that while there are inherent differences between the datasets themselves, it does appear that personality is projected in a fundamentally different way between corpora. The YouTube dataset has also been used in the Workshop on Computational Personality Recognition 2014 10. The goal of the workshop was to allow participants to compare the performance and quality of different approaches in personality recognition tasks, as well as defining the state-of-the-art. In this paper, we also use this dataset in our experiments.

\section{Datasets}

Analyses presented in this paper employ three datasets collected from the most popular social media platforms (i.e., Facebook, Twitter and YouTube). All of those datasets are available to other researchers and hence could be used to benchmark new methods and approaches. Besides for their availability, we choose these three datasets for their differences in size, users, and approach of labeling with personality scores to obtain the ground truth data.

Besides the datasets that we use in this study, there are a few golden standard datasets which are publicly available, such as the essay dataset collected by Mairesse et al. 36 and the mobile personality dataset collected by Aharoni et al. 1. However, these datasets are not social media datasets, thus we do not leverage them in this study.

There are not many golden standard datasets from social media platforms available for the personality prediction task. The main reason is that gathering labeled data is time-consuming and expensive. So far, two approaches have been used to collect personality scores. The first approach requires participation of users to provide self-reported personality via answering questionnaires. This approach has been used to gather labels for the Facebook and Twitter datasets that we use in this study. Another approach is by asking other users for their opinion regarding the personality of a user. Unlike many tasks in natural language processing where labeling data by using human resources is accurate and straightforward, assigning personality scores is a challenging task for non-experts. Using questionnaires to 
Table 2 (Table on the left) Characteristics of 3731 users in the myPersonality dataset. (Table on the right) Mean and Standard Deviation of Big Five personality scores of 3731 users (range $[1,5])$.

\begin{tabular}{|l||c|c|}
\hline & Female & Male \\
\hline \# Users & 1492 & 2239 \\
Average age & 25 & 25 \\
Avg Network size & 311 & 309 \\
Avg \# Likes & 183 & 184 \\
Avg \# Diads & 219 & 227 \\
Avg \# Education & 2 & 2 \\
Avg \# Status Updates & 176 & 185 \\
Avg \# Groups & 34 & 34 \\
\hline \hline
\end{tabular}

\begin{tabular}{|c||c|c|}
\hline Personality & Mean & Std Dev \\
\hline Extroversion & 3.60 & .81 \\
Openness & 3.90 & .66 \\
Agreeableness & 3.60 & .70 \\
Conscientiousness & 3.50 & .74 \\
Neuroticism & 2.73 & .80 \\
\hline
\end{tabular}

collect perceived personality scores can ease the task, however judging the personality of another person by employing the written or spoken text is a challenging task. even for experts. Collecting personality scores of users via face-to-face interactions or observing each other's behavior is somewhat easier; our YouTube Vlogger dataset is labeled in this way. The rest of this section provides a detailed description of the datasets that we use in this study.

\subsection{MyPersonality: Facebook Dataset}

MyPersonality [52] was a popular Facebook application introduced in 2007 allowing its users to take a number of psychometric tests, including a standard Five Factor Model questionnaire 21. Users received feedback on their scores and could optin to donate their scores and Facebook profile data to research. Data for over 6 million myPersonality users is available to researchers at: http://mypersonality.org/. It contains scores on more than 20 psychological tests, demographic profiles, and Facebook profile data including status updates, Likes, social networks, views, work and education history and much more.

The sample that is used in this work includes 3731 users who chose English as their default language and has the following information available: age, gender, personality scores, Facebook activities (i.e., counts of Likes; counts of status updates posted by the user; counts of education; counts of diads from the friendship diads table of the user; counts of group memberships for the user; and network size or number of friends of the user); and at least one status update.

Since our goal is to infer the Big Five personality scores for a given user, we identify a user with his or her set of available status updates which are treated as one text per user, his or her demographic features and Facebook activities. The Big Five personality scores for each user are available in the range of $[1,5]$. Table 2 provides details about this dataset's characteristics.

Note that the sample that we use is not the largest possible sample from the myPersonality data consisting of users with all the mentioned information. We randomly selected a sub-sample which is large enough for analysis (an order of magnitude larger than the YouTube dataset, which we will discuss next), while at the same time small enough to process with the tools that we leverage in this study. Investigating tools for big data analysis is out of the scope of this paper, thus we leave it for our future work. 


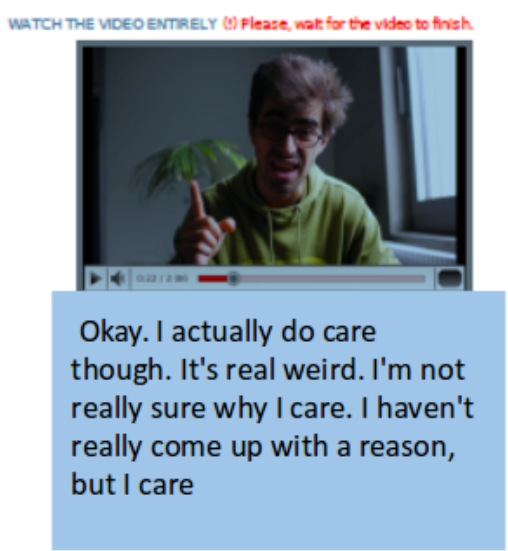

Fig. 1 An example of an excerpt from a vlog transcript.

Table 3 (Table on the left) Characteristics of the 404 users in the YouTube vloggers dataset. (Table on the right) Mean and standard deviation of the perceived Big Five personality scores of the users (range $[1,7]$ ).

\begin{tabular}{|l||c|}
\hline \multicolumn{1}{|c||}{} & Characteristics \\
\hline \# users & \# Female -210 \\
& \# Male -194 \\
\hline \# AV & \# Audio -21 \\
features & \# Video - 4 \\
\hline Transcripts & 10K unique words \\
& 240K word tokens \\
& Avg 595 words/transcript \\
\hline \hline
\end{tabular}

\begin{tabular}{|c||c|c|}
\hline Personality & Mean & Std Dev \\
\hline Extroversion & 4.62 & .98 \\
Openness & 4.66 & .72 \\
Agreeableness & 4.68 & .88 \\
Conscientiousness & 4.50 & .77 \\
Emotional Stability & 4.77 & .80 \\
\hline \hline
\end{tabular}

\subsection{YouTube Vlog Dataset}

A video blog or video log, usually abbreviated as vlog, is the video form of a blog. Vloggers explicitly show themselves in front of a webcam, talking about a variety of topics including personal issues, politics, movies, books, etc. Figure 4.2 shows an excerpt from the transcript of a vlog. The YouTube Vlog dataset ${ }^{1}$ that we use in this study was collected by Biel et al. in 2011 [6,7, and consists of 404 vlogs. For each vlog, 25 audio-video features are available, as well as a raw text speech transcript corresponding to the full video duration, the gender of the vlogger, and personality impression scores. Table 3 provides details about this dataset's characteristics and personality scores mean and standard deviation.

The personality impressions consist of Big Five personality scores that were collected using Amazon's Mechanical Turk (MTurk) crowd sourcing platform and the Ten-Item Personality Inventory (TIPI). MTurk annotators watched one-minute slices of each vlog, and rated impressions using a personality questionnaire. The Big Five personality impression scores are available for each user over all the five traits in the range of $[1,7]$.

\footnotetext{
1 https://www.idiap.ch/dataset/youtube-personality
} 
The audio-video features were automatically extracted from the conversational excerpts of the vlogs and aggregated at the video level. The video features were extracted from the vloggers body activities and include 4 features: the entropy, median, and center of gravity in horizontal and vertical dimensions. The 21 audio features include speaking time, length of the speaking segments, number of speaking turns, voicing rate, ratio of looking while speaking, ratio of looking while not speaking, and multimodal ratio, in addition to mean and standard deviation of speaking energy, pitch, looking time, length of the looking segments, number of looking turns, proximity to the camera, and vertical framing. For more details we refer to 6 .

\subsection{Twitter Dataset}

The Twitter dataset consists of a small set of 102 Twitter users, labeled with goldstandard self-assessed personality types in the range of $[-0.5,0.5]$. Users have been recruited by means of a Twitter advertising campaign in different languages and their personality types have been assessed with the 10-item personality test (BFI10) [49, which is available in the selected languages ${ }^{2}$ In addition to personality types, we collected age and gender of the Twitter users, and a set of other metadata about them. Since our Twitter dataset is multi-lingual, we first detect English speaking users with a language detector. The sample we use in the remainder of this paper includes the 44 English speaking users. For each user we have the age and gender, in addition to their tweets. The statistics of the data that we collected are reported and described in Table 4.

Table 4 (Table on the left) Characteristics of the 102 users in the Twitter dataset. (Table on the right) Mean and standard deviation of the self-reported Big Five personality scores of the users (range $[-0.5,0.5]$ ).

\begin{tabular}{|c||c|}
\hline & Info \\
\hline \#Users & 44 \\
\#Words & $30 \mathrm{~K}$ tokens \\
Average tweets per user & 19 \\
\#Males & 20 \\
\#Females & 24 \\
Average age & 27 \\
\hline
\end{tabular}

\begin{tabular}{|c||c|c|}
\hline Personality & Mean & Std Dev \\
\hline Extroversion & .16 & .18 \\
Openness & .10 & .24 \\
Agreeableness & .14 & .16 \\
Conscientiousness & .11 & .17 \\
Emotional Stability & .23 & .19 \\
\hline
\end{tabular}

The Twitter datasets from previous works that are mentioned in Section 3 are not publicly available and we do not have access to any of them. The Twitter dataset that we use in our study has recently become available as part of the PAN2015 competition ${ }^{3}$. The reason that it is small is because manually labeling text (tweets) with personality scores to obtain ground truth data is expensive and to the best of our knowledge, no other publicly available datasets of tweets exist that have been labeled with personality scores.

\footnotetext{
2 https://www.ocf.berkeley.edu/ johnlab/bfi.htm

3 http://www.uni-weimar.de/medien/webis/events/pan-15
} 


\section{Methodology}

\subsection{Extracted Features}

We extracted a wide variety of linguistic and emotional features from the three datasets that we use in this study. Pychological studies [36] show that there exist links between linguistic features (extracted from text and conversations) and users' personality traits. This finding is demonstrated by the correlations between features such as acoustic parameters, lexical categories, and n-grams on one hand, and the personality classes on the other hand 44. As a result, it has become increasingly popular to use language in social media for predicting personality. These findings motivate the choice of the following Linguistic Features extracted from text that we use in our experiments. In the rest of this section, when we refer to document, we mean the combination of all the status updates of a user in the case of the Facebook dataset, the combination of all tweets of a user in the case of the Twitter dataset and the transcript of a vlog for the case of the YouTube dataset.

- LIWC: the Linguistic Inquiry and Word Count tool, known as LIWC, is wellknown text analysis software which is widely used in psychology studies 44. Using the LIWC tool, we extracted $\mathbf{8 1}$ features from each document including features related to standard counts (e.g., word count), psychological processes (e.g., the number of anger words such as hate and annoyed in the document), relativity (e.g., the number of verbs in the future tense), personal concerns (e.g., the number of words that refer to occupation such as job and majors), and linguistic dimensions (e.g., the number of swear words). For a complete overview of the features, we refer to 53 .

- NRC: NRC is a lexicon that contains more than 14,000 distinct English words annotated with 8 emotions (anger, fear, anticipation, trust, surprise, sadness, joy, and disgust), and 2 sentiments (negative, positive) [37]. For each document we counted the number of words in each of the 8 emotion and 2 sentiment categories, resulting in $\mathbf{1 0}$ features per document. The NRC Emotion Lexicon has been used in other works for the task of personality predictions, e.g. 38 and [15. The underlying rationale for including emotional features (NRC) is that people with different personality traits will express themselves differently and, hence, will use different words (phrases) and emotions (such as anger and joy). A relation between emotions and personality traits has been observed in past research as well 14.

- MRC: MRC is a psycholinguistic databas ${ }^{4}$ which contains psychological and distributional information about words. The MRC database contains 150,837 entries with information about 26 properties (e.g., the number of syllables in the word, the number of letters, etc.), although not all properties are available for every word. Using MRC we generated $\mathbf{1 4}$ features for every document by adding the MRC-scores for each word in the document. Extracted features are: number of letters in the word (Nlet), number of phonemes in the word (Nphon), number of syllables in the word (Nsyl), Kucera and Francis written frequency (KF freq), Kucera and Francis number of categories (KF ncats), Kucera and Francis number of samples (KF nsamp), Thorndike-Lorge frequency (TL freq), Brown verbal frequency (BROWN freq), Familiarity (Fam),

\footnotetext{
4 http://www.psych.rl.ac.uk/User_Manual_v1_0.html
} 
concreteness (Conc), imagery (Imag), mean Colerado Meaningfulness (Meanc), mean Pavio Meaningfulness (Meanp), and age of acquisition (Aoa). MRC features used in previous studies such as 17 showed that there is a significant correlation between Extroversion and concreteness features, as well as between Conscientiousness and words expressing insight, longer words (Nphon, Nlet, Nsyl and Sixltr), and words that are acquired late by children (Aoa) in the MRC database.

- SentiStrength: SentiStrength ${ }^{5}$ assigns to each text a positive, negative and neutral sentiment score on a scale of 1 (no sentiment) to 5 (very strong sentiment). Texts may be simultaneously positive, negative and neutral. We used SentiStrength to compute 2 sentiment scores (2 features) for every document. There are different ways to get the output from SentiStrength. For this study we chose "dual", in which for each given text we get two values corresponding to negative and positive sentiment, and the neutral score can be calculated by summing these two numbers. We disregarded the neutral score in our study. Many studies have successfully exploited emotion and sentiment features in personality prediction tasks such as [10,15].

- SPLICE: We used SPLICE ${ }^{6}$ (Structured Programming for Linguistic Cue Extraction) to extract 66 linguistic features, including cues that relate to the positive or negative self evaluation of the speaker (e.g., I'm able, don't know), complexity and readability scores. SPLICE features have also been used in a number of psychological studies and personality prediction tasks including [15].

For the Facebook dataset, we extracted features from one textual document file per user. The complete list of the extracted features from the Facebook dataset includes the demographic features, i.e., age and gender, the Facebook activity features as explained in Table 2, such as the number of likes and status updates, and the linguistic features except for the NRC features. For the YouTube dataset, in addition to the given audio/video and gender features, for each vlogger we extracted all the linguistic features from the vlogs' transcripts. And finally, similar to the Facebook dataset, for the Twitter dataset, we have the age and gender of users and we extracted all the linguistic features, except for the NRC features, from the users' tweets.

The NRC features are not extracted from the Facebook statuses and tweets. Emotion is a momental feeling with respect to an object, person, event, or situation. As a consequence, people express a variety of different emotions over a period of time. Since we combine all status updates or tweets of a user to extract linguistic features, extracting NRC features without considering the context is irrelevant.

In this study, we extract dictionary-based linguistic features, also known as closed-vocabulary approaches, to compare the predictive ability of features across different social media platforms. Open-vocabulary linguistic features for the task of personality prediction have been studied as well in previous work, with promising results such as in [51. Examples of open-vocabulary features are n-grams, clustered groups of semantically related words (e.g., latent Dirichlet allocation (LDA) topics), and differential language analysis (i.e., DLA).

\footnotetext{
5 http://sentistrength.wlv.ac.uk

6 http://splice.cmi.arizona.edu
} 
Table 5 Pearson product-moment correlation results among personality scores on five traits: Extroversion (Extr), Agreeableness (Agr), Conscientiousness (Cons), Emotional Stability (Ems) vs. Neuroticism (Neu), and Openness (Open) on the Facebook dataset, YouTube vloggers dataset and Twitter dataset. Significant correlations $(p<.05)$ among the personality scores are indicated in bold.

\begin{tabular}{|c|c|c|c|c|c|}
\hline \multicolumn{6}{|c|}{ Facebook } \\
\hline & Extr & Agr & Cons & Neu & Open \\
\hline Extr & 1.00 & & & & \\
\hline Agr & .17 & 1.00 & & & \\
\hline Cons & .16 & .18 & 1.00 & & \\
\hline Neu & -.32 & -.33 & -.28 & 1.00 & \\
\hline Open & .14 & .04 & -.01 & -.05 & 1.00 \\
\hline \multicolumn{6}{|c|}{ YouTube } \\
\hline & Extr & Agr & Cons & Ems & Open \\
\hline Extr & 1.00 & & & & \\
\hline Agr & .02 & 1.00 & & & \\
\hline Cons & -.03 & .38 & 1.00 & & \\
\hline Ems & .06 & .69 & .54 & 1.00 & \\
\hline Open & .56 & .29 & .26 & .30 & 1.00 \\
\hline \multicolumn{6}{|c|}{ Twitter } \\
\hline & Extr & Agr & Cons & Ems & Open \\
\hline Extr & 1.00 & & & & \\
\hline Agr & 0.27 & 1.00 & & & \\
\hline Cons & 0.01 & 0.1 & 1.00 & & \\
\hline Ems & 0.46 & 0.34 & 0.15 & 1.00 & \\
\hline Open & -0.05 & -0.06 & 0.1 & 0.05 & 1.00 \\
\hline
\end{tabular}

Unlike open-vocabulary approaches, the quality and processing time of the features extracted by the dictionary-based approaches do not depend on the size of the data. However, one limitation of using dictionary-based linguistic features for the task of personality prediction in social media is the dynamic and noisy structure of these platforms. Users in social media tend to use informal language which contains language errors, misspelled words and newly defined terms and phrases. Thus, improving the performance of the dictionary-based approaches on user generated texts in social media is an open path to explore.

\subsection{Regression Approaches}

Regression is the task of predicting a continuous, real valued output from a set of predictors. As the name implies, univariate regression refers to estimating a regression model with one dependent variable (one outcome), while multivariate regression refers to building a regression model with more than one dependent variable (several outcomes).

The results in Table 5 indicate a clear correlation among different personality trait scores in the YouTube, Facebook and Twitter datasets. The dependency among different personality scores makes personality score prediction a good candidate for multivariate regression, where the dependencies between the target variables are taken into account to make a combined prediction.

Formally, univariate/multivariate regression addresses this problem: let $\mathcal{F}$ be the input space consisting of vectors with values for $m$ features, $f_{1}, f_{2}, \ldots, f_{m}$, and 
let $\mathcal{T}$ be the output space consisting of vectors with values for $n$ target variables $t_{1}, t_{2}, \ldots, t_{n}$. The goal of a multivariate regression algorithm is to learn a model $\mathbf{M}: \mathcal{F} \rightarrow \mathcal{T}$ that minimizes the prediction error over a training set.

In this study, $n=5$ (where $t_{1}$ is Extroversion, $t_{2}$ is Agreeableness, $t_{3}$ is Conscientiousness, $t_{4}$ is Emotional Stability/Neuroticm and $t_{5}$ is Openness). Using this formulation, the univariate and multivariate regression algorithms that we use in this paper are 54]:

1. Single-Target (ST): In ST, for each target variable $t_{i}$, a single model $M_{i}$ : $\mathcal{F} \rightarrow \mathcal{T}_{i}$ is trained that maps a vector from the input space $\mathcal{F}$ to a value in $\mathcal{T}_{i}$, which is the range of variable $t_{i}$. The results of the desired multi-target model $\mathbf{M}$ are comprised of the outcomes of the single-target models.

2. Multi-Target Stacking (MTS): MTS consists of two steps. In the first step, $n$ single-target models are used as in ST, however, MTS includes an additional step where the input space for each target variable is expanded by the predicted results of the other target variables ( $n-1$ predicted values) from step one. Let $t_{1}^{\prime}, t_{2}^{\prime}, \ldots, t_{n}^{\prime}$ be the prediction results from the first step, then, for example, the input space for $t_{1}$ in step two is $\left[f_{1}, f_{2}, \ldots, f_{m}, t_{2}^{\prime}, t_{3}^{\prime}, \ldots, t_{n}^{\prime}\right]$.

3. Multi-Target Stacking Corrected (MTSC): In MTSC, an internal crossvalidation sampling technique is used to avoid over-estimation of the training set. In MTSC, by using $k$-fold sampling, the prediction results of $\frac{k-1}{k} \%$ of the whole training set are used to expand the input space in the second step as in MTS. In this study we use $k=10$.

4. Ensemble of Regressor Chains (ERC): The idea behind ERC is chaining single-target regression models. By choosing an order for the target variables (e.g., $O=\left(t_{1}, t_{2}, \ldots, t_{n}\right)$ ), the learning model for each target variable $t_{j}$ relies on the prediction results of all target variables $t_{i}$ which appear before $t_{j}$ in the list. For the first target variable, a single-target regression model as in ST predicts the value, then the input space for the next target variable is extended with the prediction results of the previous one and so on. Since in this model the order of the chosen chain affects the results, the average prediction result of $r$ different chains (in our study we choose $r=10$, as is typically done) for each target variable is used as the final prediction result.

5. Ensemble of Regressor Chains Corrected (ERCC): The difference between $E R C$ and $E R C C$ is similar to that between $M T S$ and $M T S C$, i.e., the use of $k$-fold sampling to increase the reliability of the predictions based on the training set. In this study we use $k=10$.

6. Multi-objective random forest (MORF): $M O R F$ is a random forest ensemble technique of multi-objective decision trees (MODT). Each MODT is a multi-target regression model that predicts multiple target variables at once. MODT models are instantiations of predictive clustering trees (PCTs) that are used for multi-objective prediction [8]. The PCTs algorithm and standard decision trees differ in the way they treat the variance and the prototype functions. In PCTs, the variance and the prototype functions are treated as parameters, and they are instantiated towards a given prediction task for computing the leaf labels. For multi-objective regression trees, the variance is computed as the sum of the variances of the target variables $\left(t_{i}\right)$. That is, $\operatorname{Var}(E)=\sum_{i=1}^{n} \operatorname{Var}\left(t_{i}\right)$, where $E$ is a set of training examples, and each leaf's prototype is the vector mean of the target vectors of its training exam- 
ples. Multi-objective random forests $(M O R F)$ have shown better predictive performance than their counter ensemble methods like bagging for MODT 30.

Note that ST does not leverage the prediction result for one personality trait to make a prediction for another, while all other algorithms (MTS, MTSC, ERC, $E R C C$ and $M O R F$ ) do in one way or another. To get the results for $S T, M T S$, $M T S C, E R C, E R C C$ and $M O R F$ we used the implementation of these algorithms in Mulan 7 The base learner of these algorithms in Mulan (except MORF, as explained above) is the Weka decision tree algorithm. For further information we refer to [54. For the $E R C$ and $E R C C$ models we choose 10 randomly selected chains and for MORF we use an ensemble size of 100 trees. For the rest of the parameters we use the suggestions in 30 .

We also use the R software environment 48, to implement $S T$ and $M T S$ with a support vector machine regressor with radial kernel as a base learner. In the remainder of this paper we mention the base learner in parentheses after the approach name to make it clear which base learner is used, for example, MTS $(S V M)$ refers to the Multi-Target Stacking approach with a support vector machine regressor as a base learner. In the case of $S V M$, we tried different kernels, namely radial, linear and polynomial, and tuned the parameters based on the training set. Since we obtained the best results with a radial kernel, all results presented throughout this paper that are based on a SVM as base leaner rely on a radial kernel.

\subsection{Evaluation Approaches}

We evaluate the results based on Root Mean Squared Error (RMSE) and Coefficient of Determination $\left(R^{2}\right)$. RMSE measures the difference between the predicted values by a model and the observed values. RMSE ranges from 0 to $\infty$ where lower values signify better models. RMSE can be described by the following formula:

$$
R M S E=\sqrt{\frac{\sum_{t=1}^{n}\left(y_{o b s}^{t}-y_{\text {pred }}^{t}\right)^{2}}{n}}
$$

where $y_{\text {obs }}^{t}$ and $y_{\text {pred }}^{t}$ are the observed and predicted scores for instance $t$ (where $t=1 \ldots n)$ and $n$ is the sample size.

$R^{2}$ is the ratio of the model's absolute error and the baseline mean predicted scores. It is expressed as:

$$
R^{2}=100 \times\left(1-\frac{\sum_{t=1}^{n}\left(y_{o b s}^{t}-y_{p r e d}^{t}\right)^{2}}{\sum_{t=1}^{n}\left(y_{o b s}^{t}-\hat{y}_{o b s}\right)^{2}}\right)
$$

where $y_{o b s}^{t}$ and $\hat{y}_{\text {obs }}$ are respectively the observed scores and their mean, and $y_{\text {pred }}^{t}$ are the predicted scores by the model. $R^{2}$ measures the relative improvement of the mean squared error using the automatic predictor compared to the average baseline. Positive values indicate that the model accounts for a greater proportion

\footnotetext{
7 http://mulan.sourceforge.net/
} 
of the variance in the data thus outperforming the constant average baseline. Negative values indicate that variation in the data accounted for by the model is worse than the baseline score, thus not outperforming the baseline.

\section{Experimental Results}

In this section, we present the details of the experiments and the results of personality prediction using our three social media datasets.

\subsection{Correlation Results}

We perform pair-wise correlation analysis between the extracted features and personality scores for all three datasets. In particular we use the non-parametric Spearman rank correlation to compute the correlations in the YouTube and Twitter datasets due to the non-normal and highly skewed nature of the distribution of individual features. For the Facebook dataset we use the parametric Pearson correlation when reporting the correlations. For computing Spearman and Pearson correlations between the features and the five personality scores, we use the $\mathrm{R}$ software environment 48.

Table 6 contains a summary of the most important correlation results across all three social media datasets. All the presented correlation results are significant with $p<0.058$

The demographic features age and gender have a significant correlation with personality scores across all three datasets. Following a commonly adopted encoding approach, in our experiments, gender equal to 1 indicates female users and 0 indicates male. In fact correlation with the gender feature is simply a comparison of the means of personality scores for men and women. An appropriate approach to calculate this association is point-biserial correlation which is mathematically equivalent to the Pearson correlation by using $0 / 1$ values. Thus, we use the Pearson correlation for finding the relations among personality traits and gender for all three datasets.

There is a positive relation (0.06) between gender and the Agreeableness personality trait on Facebook. However, the relation is negative $(-0.24)$ in case of the YouTube dataset and Twitter dataset (-0.18). This means that for female Facebook users, the mean personality score for Agreeableness will be higher than men, but lower in case of YouTube and Twitter users. In addition, age has a similar correlation (0.04) with the Emotional stable and Agreeableness personality scores.

In case of linguistic inquiry and word count (LIWC) features, six features were found to be common and significantly correlated across the three datasets. Similar to demographic features, these LIWC features exhibit different relations depending on the dataset type. For example, the word count $(W C)$ shows a positive relation with the Agreeableness personality score in the Facebook (0.02) and Twitter (0.31) datasets, but is negatively related in the YouTube dataset $(-0.11)$.

\footnotetext{
8 We compute the correlation among all features and personality traits and find the significant correlated features. The full list of features and their correlation scores can be downloaded from the supplementary materials of this manuscript.
} 
Table 6 Common significantly $(p<.05)$ correlated features with personality traits. The personality traits: Extroversion (Extr), Agreeableness (Agr), Conscientiousness (Cons), Emotional Stability (Ems) vs. Neuroticism (Neu), and Openness (Open), across Facebook, YouTube and Twitter datasets. The significant features after Bonferroni-correction (with $p<.01)$ are typeset in bold.

\begin{tabular}{|c|c|c|c|c|}
\hline Feature & Trait & Facebook & YouTube & Twitter \\
\hline \multicolumn{5}{|c|}{ Demographics } \\
\hline Gender & Agr & 0.06 & -0.24 & -0.18 \\
\hline Age & Ems/Neu & 0.04 & - & 0.32 \\
\hline Age & Agr & 0.04 & - & 0.41 \\
\hline \multicolumn{5}{|c|}{ LIWC } \\
\hline WC (word count) & Agr & 0.02 & -0.11 & 0.31 \\
\hline negate & Cons & -0.03 & -0.22 & -0.42 \\
\hline health & Ems/Neu & -0.04 & -0.11 & 0.31 \\
\hline assent & Extr & 0.03 & 0.17 & 0.33 \\
\hline motion & Open & -0.02 & 0.11 & -0.31 \\
\hline leisure & Ems/Neu & 0.04 & 0.12 & 0.43 \\
\hline \multicolumn{5}{|c|}{ MRC } \\
\hline $\mathrm{AOA}$ & Cons & 0.04 & 0.16 & 0.33 \\
\hline NLET & Agr & 0.05 & -0.11 & 0.31 \\
\hline \multicolumn{5}{|c|}{ SPLICE } \\
\hline num Adjectives & Agr & 0.04 & -0.13 & 0.30 \\
\hline SWN Positivity & Agr & 0.05 & 0.19 & 0.32 \\
\hline SWN Negativity & Agr & -0.02 & -0.20 & 0.37 \\
\hline
\end{tabular}

There were only two features - age of acquisition rating $(A O A)$, and number of letters (NLET) from the MRC psycholinguistic database that are common and have a significant correlation with the personality scores across the three datasets. Both features show a positive relation with Conscientiousness and Agreeableness personality scores for the Facebook (0.04 and 0.05) and Twitter (0.33 and $0.31)$ datasets. But, a negative correlation is found between the number of letters $(N L E T)$ and the Agreeableness personality score in the YouTube dataset.

Finally, four features from SPLICE were found to be highly correlated and common among the three datasets. Interestingly, all these features were only correlated to the Agreeableness personality score. The relation was positive in the Facebook and Twitter datasets, but mostly negative in the YouTube dataset.

Furthermore, to avoid type 1 error of multiple testings, we apply Bonferroni correction with $(p<.01)$. To have a fair comparison among the datasets, we only consider the common non zero features, thus we identify the correlations among 161 common features and 5 personality traits between three datasets. By adjusting the p-values, the number of significant correlations among features and personality traits are decreased. For the case of the Twitter dataset the number of significant features reduces from 51 significant correlated features to 11, for the case of the YouTube dataset the number of significant correlated features drops from 231 to 141, and finally for the case of the Facebook dataset the number of significant correlated features cuts down from 240 to 164 significant correlations.

The relation between the sample size and number of correlated features is addressed in 51] for the personality prediction in Facebook. Similarly, we discover a direct relation between the population size and number of correlated features, i.e., we find 11 significant correlations for the case of the Twitter dataset with only 44 examples, 140 significant correlations for the case of YouTube with 404 
samples and 164 correlations for the Facebook dataset with 3731 users. Since the population size affects the number of features, by adjusting the correlations, we do not find any common significant correlated features among all three datasets.

Overall, two key observations can be made from the results in the correlation Table 6. First, not all features are common and significantly correlated to the personality scores. For instance, among the 81 LIWC features, only six features were found to be significantly correlated $(p<.05)$ and common in all three datasets. Second, features can have a different relation with the personality score depending on the dataset. In one dataset, a feature can be positively related to a personality score (e.g., gender for Agreeableness in Facebook), while the same feature may have a negative correlation in a different dataset (e.g., gender for Agreeableness in YouTube). This suggests that it may not be possible to generalize the correlation between features and personality traits, as this may vary depending on the social media platform.

\subsection{Regression Models}

In this section, by using the univariate and multivariate regression formulations that we described in section 5.2 , we explore different approaches to computational personality recognition of social media users. We predict personality on a continuous scale which is common in psychology studies. While we predict the perceived personality scores from spoken text (transcripts from video) in the YouTube vloggers dataset, we predict the self-reported personality scores from written text as status updates and tweets from the Facebook and the Twitter datasets, respectively.

The experimental results using feature selection are presented in Section 6.3 and then results of applying different univariate and multivariate regression formulations are presented in Section 6.4. All results are based on 10-fold crossvalidation, where folds are randomly sampled from the data.

Throughout this section, we use letter codes for different regressors as described in Table 7.

Table 7 Regressors and the corresponding letter codes.

\begin{tabular}{|c|c|c|}
\hline Regressor & Base learner & Letter Code \\
\hline \multicolumn{3}{|c|}{ Univariate Regressors } \\
\hline Single-Target & Decision tree & ST (DT) \\
\hline Single-Target & Support vector machine & ST (SVM) \\
\hline \multicolumn{3}{|c|}{ Multivariate Regressors } \\
\hline Multi-Target Stacking & Decision tree & MTS (DT) \\
\hline Multi-Target Stacking & Support vector machine & MTS (SVM) \\
\hline Multi-Target Stacking Corrected & Decision tree & $\operatorname{MTSC}(\mathrm{DT})$ \\
\hline Ensemble of Regressor Chains & Decision tree & $\mathrm{ERC}(\mathrm{DT})$ \\
\hline Ensemble of Regressor Chains Corrected & Decision tree & $\operatorname{ERCC}(\mathrm{DT})$ \\
\hline Multi-objective random forest & Multi object decision tree & MORF \\
\hline
\end{tabular}




\subsection{Experiments Using Feature Selection}

Previous studies with regard to personality prediction suggest that feature selection can improve the accuracy of learning algorithms 16. Feature subset selection is the process of identifying relevant features and removing irrelevant and redundant features before training of the model. It has been shown that feature subset selection enhances the performance of learning algorithms by reducing the hypothesis search space and/or reducing the storage or processing requirement [24].

The main focus of our study w.r.t. feature selection and feature correlation analysis in the paper is on understanding and assessing the impact of individual input features on personality prediction. Our goal is to identify features that are most predictive and relevant to the target variable. We have not measured the correlation among input features themselves. While we acknowledge that that would be interesting as it might lead to regressors with higher accuracy and/or a reduced feature space, we consider that beyond the scope of this paper.

Our incentive for performing feature selection based on correlation analysis is that it is a so-called filter based approach. Unlike wrapper or embedded feature selection approaches [22, filter based feature selection does not depend on the underlying learner, therefore our feature analysis results are general and not tied to a specific learner. Finally, we acknowledge that there are many feature construction methods for dimensionality reduction such as basic linear transforms of the input variables (e.g., PCA [29]) that can improve the performance of the learner, but as stated above, we consider this to be beyond the scope of this paper.

We perform experiments by selecting different feature sets. We first grouped features based on their categories and then the relevant subset of features for each category is identified by conducting correlation analysis as explained in Section 6.1. Hence, to select features from each category, we choose the significantly correlated features with a trait with $p<0.05$. Next, for each feature category, we perform personality score prediction based on the selected features, using singletarget regression with SVM as the base learner. All results presented in Table 8 are averaged over 10-fold cross-validation. In every fold, the correlated features are calculated based only on the training examples, hence the correlated features may differ from one fold to another. The results are specific to each social media platform.

In the case of Facebook, we leverage six feature sets in addition to their corresponding correlated feature sets. By "correlated feature set" we mean the subset of features that was found to be correlated with the personality trait at hand. Results which are presented in Table 8 indicate that Facebook activities and demographics of a user are better predictors in learning the personality of a user compared to their user generated texts, i.e., extracted features from their status updates. For the case of predicting scores for Extroversion and Neuroticism, using only this feature set is enough to get the lowest RMSE score. However, for the traits Agreeableness, Conscientiousness and Openness, in addition to this feature category, textual features from the combined status updates improve the performance and lead to the lowest RMSE. Among the five different feature sets (i.e., except for the combination of all features as one feature set $A l l$ ) that we extracted for this dataset, users' activities and demographics in addition to LIWC features produce the lowest RMSE for predicting personality scores for all five traits. 
Table 8 RMSE Comparison of three datasets including 3731 Facebook users, 404 YouTube vloggers, and 44 Twitter users by applying all features and correlated features under each feature set category. For each feature set category, using the correlated features in a model is shown with $\checkmark$ while a model which uses all features is marked with $\boldsymbol{X}$. The personality traits are Extroversion (Extr), Agreeableness (Agr), Conscientiousness (Cons), Emotional Stability (Ems) vs. Neuroticism (Neu), and Openness (Open). All results are based on 10-fold crossvalidation using SVM (radial kernel). In each column, significant differences $(p<.05)$ with respect to the baseline are denoted by a $*$ sign, and the lowest RMSEs are typeset in bold. The average baseline is shown with Avg.

\begin{tabular}{|c|c|c|c|c|c|c|}
\hline \multicolumn{7}{|c|}{ Facebook } \\
\hline Feature set & Correlated & $\begin{array}{c}\text { Extr } \\
\text { RMSE }\end{array}$ & $\begin{array}{c}\text { Agr } \\
\text { RMSE }\end{array}$ & $\begin{array}{c}\text { Cons } \\
\text { RMSE }\end{array}$ & $\begin{array}{c}\text { Neu } \\
\text { RMSE }\end{array}$ & $\begin{array}{c}\text { Open } \\
\text { RMSE }\end{array}$ \\
\hline Avg & & .807 & .699 & .735 & .786 & .661 \\
\hline Activity \& Demographics & $x$ & .784 & .702 & .721 & .768 & .663 \\
\hline Activity \& Demographics & $\checkmark$ & .785 & .702 & .721 & .768 & .664 \\
\hline LIWC & $x$ & .803 & .693 & .723 & .779 & .652 \\
\hline LIWC & $\checkmark$ & .806 & .693 & .725 & .782 & .657 \\
\hline SentiStrength & $x$ & .807 & .697 & .734 & .786 & .664 \\
\hline SentiStrength & $\checkmark$ & .810 & .703 & .737 & .787 & .660 \\
\hline $\mathrm{MRC}$ & $x$ & .811 & .700 & .730 & .787 & .663 \\
\hline MRC & $\checkmark$ & .809 & .699 & .729 & .785 & .661 \\
\hline SPLICE & $x$ & .807 & .699 & .730 & .785 & .664 \\
\hline SPLICE & $\checkmark$ & .810 & .701 & .736 & .788 & .665 \\
\hline All & $x$ & .791 & .695 & .717 & .773 & .651 \\
\hline All & $\checkmark$ & .786 & .692 & .719 & .770 & .653 \\
\hline \multicolumn{7}{|c|}{ YouTube } \\
\hline & & $\begin{array}{c}\text { Extr } \\
\text { RMSE }\end{array}$ & $\begin{array}{c}\text { Agr } \\
\text { RMSE }\end{array}$ & $\begin{array}{c}\text { Cons } \\
\text { RMSE }\end{array}$ & $\begin{array}{c}\text { Ems } \\
\text { RMSE }\end{array}$ & $\begin{array}{c}\text { Open } \\
\text { RMSE }\end{array}$ \\
\hline Avg & & .980 & .880 & .773 & .780 & .719 \\
\hline Gender, Audio \& Video & $x$ & $.842^{*}$ & .892 & .759 & .787 & .706 \\
\hline Gender, Audio \& Video & $\checkmark$ & $.868^{*}$ & .882 & .752 & .824 & .704 \\
\hline LIWC & $x$ & .930 & $.781^{*}$ & $.683^{*}$ & .753 & .710 \\
\hline LIWC & $\checkmark$ & .933 & $.775^{*}$ & $.695^{*}$ & .752 & .716 \\
\hline $\mathrm{NRC}$ & $x$ & .984 & $.814^{*}$ & .757 & .767 & .712 \\
\hline NRC & $\checkmark$ & 1.00 & $.816^{*}$ & .774 & .774 & .712 \\
\hline SentiStrength & $x$ & .987 & $.805^{*}$ & .758 & $.741^{*}$ & .710 \\
\hline SentiStrength & $\checkmark$ & .987 & $.815^{*}$ & .774 & .746 & .716 \\
\hline MRC & $x$ & .969 & .900 & $.743^{*}$ & .790 & .721 \\
\hline MRC & $\checkmark$ & .975 & .920 & $.746^{*}$ & .793 & .725 \\
\hline SPLICE & $x$ & .979 & .882 & .772 & .779 & .717 \\
\hline SPLICE & $\checkmark$ & .971 & .882 & .773 & .794 & .718 \\
\hline All & $x$ & .979 & .882 & .773 & .780 & .717 \\
\hline All & $\checkmark$ & $.867^{*}$ & $.773^{*}$ & $.708^{*}$ & $.742^{*}$ & .700 \\
\hline \multicolumn{7}{|c|}{ Twitter } \\
\hline & & Extr & Agr & Cons & Ems & Open \\
\hline & & RMSE & RMSE & RMSE & RMSE & RMSE \\
\hline Avg & & .179 & .159 & .175 & .198 & .236 \\
\hline Demographics & $x$ & .187 & .161 & .203 & .213 & .211 \\
\hline Demographics & $\checkmark$ & .213 & .149 & .203 & .195 & .202 \\
\hline LIWC & $x$ & .181 & .160 & .175 & .208 & .253 \\
\hline LIWC & $\checkmark$ & .181 & .160 & .175 & .288 & .253 \\
\hline SentiStrength & $x$ & .184 & .156 & .174 & .193 & .256 \\
\hline SentiStrength & $\checkmark$ & .180 & .163 & .132 & .194 & .235 \\
\hline MRC & $x$ & .180 & .164 & .170 & .192 & .236 \\
\hline MRC & $\checkmark$ & .194 & .178 & .167 & .189 & .270 \\
\hline SPLICE & $x$ & .185 & .163 & .183 & .188 & .240 \\
\hline SPLICE & $\checkmark$ & .173 & .159 & .247 & .215 & .252 \\
\hline All & $x$ & .181 & .165 & .183 & .179 & .226 \\
\hline All & $\checkmark$ & .197 & .162 & .184 & .204 & .230 \\
\hline
\end{tabular}


In the case of the YouTube vloggers, we analyze seven feature sets and their corresponding correlated ones. The audio and video features extracted from the videos, which reflect the actual behavior of the users, are better predictors compared to the linguistic features for predicting the score of Extroversion. However, for other traits, the lowest RMSEs are obtained by leveraging the content of the videos by using the linguistic features extracted from the transcripts. For the case of Agreeableness and Openness, using the combination of linguistic features and audio and video features in the learning process, resulted in the lowest RMSE. And finally, the models that use LIWC features for Conscientiousness score prediction and SentiStrength features for inferring the Emotional Stability trait show results with the lowest RMSE score. Overall, for the YouTube dataset using only LIWC features produces better prediction results compared to other feature sets.

For the case of the Twitter dataset, we use six feature sets in addition to their corresponding correlated feature sets. It is interesting that from the demographic features, using only age for inferring the Agreeableness score and only gender for predicting the Openness score outperform the average baseline while for the case of Emotional Stability, using the combination of all feature groups as one feature space led to the best performing model which also outperforms the average baseline. Textual features extracted from the tweets, in particular SPLICE features for the case of Extroversion and SentiStrength for the case of Conscientiousness, reduce the error and outperform the average baseline. For this dataset, due to the small size of the training set, the results obtained using various feature sets are very similar and choosing one feature set that outperforms other feature sets for all traits is not possible.

Overall, for all the traits in all three data sets, we find at least one feature set which outperforms the average baseline. Note that the feature selection approach only considers the significant correlated features. For feature category and and traits combinations for which no significant correlated features were found, we report the same value as in the case that all features in the feature set are used. From the results in Table 8, we can conclude that selecting features using correlation analysis mostly has little or no improvement compared to using the complete feature set.

\subsection{Experiments Using Univariate and Multivariate Regression Approaches}

Following the formulations of multiple regression approaches in Section 5.2 , the formal definition of regression learners for each dataset is presented as follows. Let $\mathcal{F}$ be the input space consisting of feature vectors. The extracted features for each dataset are different as described in Section 5.1. The Facebook feature space $\mathcal{F}^{F B}$ has 171 features, $f_{1}^{F B}, f_{2}^{F B}, \ldots, f_{171}^{F B}$, the YouTube feature space $\mathcal{F}^{Y T}$ has 199 features, $f_{1}^{Y T}, f_{2}^{Y T}, \ldots, f_{199}^{Y T}$, and finally, the Twitter feature space $\mathcal{F}^{T W}$ has 165 features, $f_{1}^{T W}, f_{2}^{T W}, \ldots, f_{165}^{T W}$.

Let $\mathcal{T}$ be the output space, containing vectors with values for 5 target variables: $t_{1}$ (Extroversion), $t_{2}$ (Agreeableness), $t_{3}$ (Conscientiousness), $t_{4}$ (Neuroticm or Emotional Stability) and $t_{5}$ (Openness). The goal of a multivariate regression algorithm is to learn a model $\mathbf{M}: \mathcal{F} \rightarrow \mathcal{T}$ that minimizes the prediction error $R M S E$ over a test set. The goal of a univariate regression algorithm is to learn five models $M_{1}: \mathcal{F} \rightarrow \mathcal{T}_{1}$ (Extroversion), $M_{2}: \mathcal{F} \rightarrow \mathcal{T}_{2}$ (Agreeableness), $M_{3}:$ 
$\mathcal{F} \rightarrow \mathcal{T}_{3}$ (Conscientiousness), $M_{4}: \mathcal{F} \rightarrow \mathcal{T}_{4}$ (Neuroticm/EmotionalStability), and $M_{5}: \mathcal{F} \rightarrow \mathcal{T}_{5}$ (Openness) that minimize the prediction error RMSE over a test set, with $\mathcal{T}_{i}$ the range of variable $t_{i}$ (for $i=1 \ldots 5$ ).

Some initial research has been done on the use of multivariate regression for personality prediction on Facebook [3, 27, YouTube [15] and Sina Microblog data [5. In the current section we investigate whether the promising trend of good results can be extended to our Facebook, YouTube and Twitter datasets. To compare the performance of different regressor approaches, we apply the same set of approaches on all three datasets. We aim to identify which approach is a better predictor for the task of personality prediction regardless of the dataset. The results of all the experiments are summarized in Table 9. All results are averaged over a 10-fold cross-validation, and to measure significant differences in prediction errors between the learned models and the baseline, we conducted two-tailed paired t-tests for the RMSE, and two-tailed single t-tests for $R^{2}$ at the $p<.05$ level.

We use two base learners in our experiments, namely a decision tree algorithm and $S V M$ algorithm. By using the whole feature space, univariate regressor $S T(D T)$ always outperforms $S T(S V M)$; similarly multivariate $M T S(D T)$ accomplishes significantly better results compared to $M T S(S V M)$. Although in many studies $S V M$ has been used successfully for inferring personality traits as a classifier or a regressor approach such as [45, 16, 39, the results presented in Table 9. which are based on three different social media datasets, indicate that the decision tree algorithm is a better predictor approach for this task.

Moreover, it can be seen from the results in Table 9 that all five algorithms (i.e., $S T(D T), \operatorname{MTS}(D T), \operatorname{MTSC}(D T), E R C(D T)$ and ERCC $(D T))$ which use the decision tree algorithm as base learner outperform (i.e., have a lower prediction error than) the average baseline model for all five personality traits. In addition, positive values for $R^{2}$ are also observed for all the algorithms which further indicates better performance than the average baseline model $\left(0 \% \leq R^{2} \leq 33 \%\right)$.

An interesting observation is that multivariate regression approaches (i.e., $\operatorname{MTS}(D T), M T S C(D T), E R C(D T)$ and ERCC $(D T))$ not always outperform the univariate approach i.e., $S T(D T)$, but most of the times they give better results. However, the differences between univariate and multivariate regressors are not significant. Overall, ERCC (DT) and MTSC (DT) outperform the other approaches across all three different datasets for all five personality predictions.

Although feature selection as suggested in many studies such as 16 can generate promising results for the task of personality prediction, using the full feature space for the results presented in Table 9 indicate that feature selection as we use in this study (Table 8 ) barely yields any advantage. Overall, $E R C C(D T)$ for all traits in the Facebook dataset, $\operatorname{MTSC}(D T)$ for YouTube, and both $E R C C(D T)$ and $M T S C(D T)$ outperform all other approaches in predicting the personality traits and yield a lower RMSE score compared to the average base line.

Finally, while Agreeableness followed by Extroversion are the easiest personality traits of YouTube vloggers to predict using the observers' score as ground truth, Extroversion followed by Conscientiousness are the best performing traits using the self-reported personality models of Facebook, and similarly Openness followed by Conscientiousness are the easiest trait to predict for self-reported personality of Twitter users. 
Table 9 Root mean square error $(R M S E)$ and Coefficient of determination $\left(R^{2}\right)$ results for personality trait prediction using univariate and multivariate regression algorithms on all 3 datasets. The personality traits are Extroversion (Extr), Agreeableness (Agr), Conscientiousness (Cons), Emotional Stability (Ems) vs. Neuroticism (Neu), and Openness (Open). All results are averaged over a 10-fold cross-validation. In each column, the lowest error and highest determination are typeset in bold. Significant differences with respect to the baseline $(p<.05)$ are marked using *. The average baseline is shown with Avg.

\begin{tabular}{|c|c|c|c|c|c|c|c|c|c|}
\hline \multicolumn{10}{|c|}{ Facebook } \\
\hline \multirow[b]{2}{*}{ Approach } & \multicolumn{2}{|c|}{ Extr } & \multicolumn{2}{|c|}{ Agr } & \multicolumn{2}{|c|}{ Cons } & Ems & \multicolumn{2}{|c|}{ Open } \\
\hline & \multicolumn{2}{|c|}{$R M S E \quad R^{2}$} & \multicolumn{2}{|c|}{$R M S E \quad R^{2}$} & \multicolumn{2}{|c|}{$R M S E \quad R^{2}$} & $R M S E \quad R^{2}$ & \multicolumn{2}{|c|}{$R M S E \quad R^{2}$} \\
\hline \begin{tabular}{l|l} 
Avg \\
\end{tabular} & \multicolumn{2}{|c|}{.807} & \multicolumn{2}{|c|}{.699} & \multicolumn{2}{|c|}{.735} & .786 & \multicolumn{2}{|c|}{.661} \\
\hline \multicolumn{10}{|c|}{ Univariate/Multivariate Regressions using Decision Tree } \\
\hline $\mathrm{ST}$ (DT) & .777 & \begin{tabular}{l|l}
7.30 \\
\end{tabular} & .691 & 2.28 & .713 & 5.90 & $\begin{array}{ll}.765 & 5.27\end{array}$ & .649 & 3.60 \\
\hline MTS (DT) & .782 & 6.10 & .698 & 0.29 & .717 & 4.84 & 3.43 & .650 & 3.30 \\
\hline $\operatorname{MTSC}(\mathrm{DT})$ & .777 & 7.30 & 690 & 2.56 & .714 & 5.63 & .763 & .649 & 3.60 \\
\hline $\operatorname{ERC}(\mathrm{DT})$ & .776 & 7.54 & .690 & 2.56 & .713 & 5.90 & 5.12 & .649 & 3.60 \\
\hline $\operatorname{ERCC}(\mathrm{DT})$ & .776 & 7.54 & 690 & 2.67 & .713 & 5.90 & 5.77 & .649 & 3.60 \\
\hline MORF & .787 & 4.90 & .693 & 1.71 & .720 & 4.04 & $.774 \quad 3.03$ & .653 & 2.41 \\
\hline \multicolumn{10}{|c|}{ Univariate/Multivariate Regressions using SVM } \\
\hline ST (SVM) & .791 & 3.93 & .695 & 1.14 & .717 & 4.84 & $\begin{array}{ll}.773 & 3.28\end{array}$ & .651 & 3.00 \\
\hline $\operatorname{MTS}(\mathrm{SVM})$ & .802 & 1.24 & .695 & 1.14 & .718 & 4.57 & .789 & .651 & 3.00 \\
\hline \multicolumn{10}{|c|}{ YouTube } \\
\hline & \multicolumn{2}{|c|}{ Extr } & \multicolumn{2}{|c|}{ Agr } & \multicolumn{2}{|c|}{ Cons } & Ems & $\mathrm{Op}$ & en \\
\hline Approach & $R M S E$ & $R^{2}$ & $R M S E$ & $R^{2}$ & $R M S E$ & $R^{2}$ & $R M S E \quad R^{2}$ & $R M S E$ & $R^{2}$ \\
\hline Avg & .980 & & .880 & & .773 & & .780 & .719 & \\
\hline & & nivariate & Multive & ariate $\mathrm{R}$ & gression & is using & Decision Tree & & \\
\hline $\mathrm{ST}(\mathrm{DT})$ & $.858^{*}$ & $23.35^{*}$ & $.724^{*}$ & $32.31^{*}$ & $.692^{*}$ & $19.86^{*}$ & .696 $^{*} 20.38^{*}$ & .695 & 6.56 \\
\hline MTS (DT) & $.862^{*}$ & $22.63^{*}$ & $.722^{*}$ & $32.69^{*}$ & $.696^{*}$ & $18.93^{*}$ & $.711^{*} 16.91^{*}$ & .690 & 7.90 \\
\hline $\operatorname{MTSC}(\mathrm{DT})$ & $.850^{*}$ & $24.80^{*}$ & $.720^{*}$ & $33.06^{*}$ & $.690^{*}$ & $20.32^{*}$ & $19.46^{*}$ & 690 & 7.90 \\
\hline $\operatorname{ERC}(\mathrm{DT})$ & $.850^{*}$ & $24.80^{*}$ & $.740^{*}$ & $29.29^{*}$ & $.700^{*}$ & $17.35^{*}$ & $19.46^{*}$ & .690 & 7.9 \\
\hline $\operatorname{ERCC}(\mathrm{DT})$ & $.853^{*}$ & $24.23^{*}$ & $.721^{*}$ & $32.87^{*}$ & $.690^{*}$ & $20.32^{*}$ & $20.15^{*}$ & .693 & 7.10 \\
\hline MORF & .908 & 14.15 & $.771^{*}$ & $23.24^{*}$ & $.699^{*}$ & $18.23^{*}$ & $.719^{*} \quad 15.0^{*}$ & .703 & 4.40 \\
\hline & & Univa & iate $/ \mathrm{Mu}$ & ultivaria & Regres & ssions $\mathrm{u}$ & ing SVM & & \\
\hline ST (SVM) & .979 & .204 & .882 & -.45 & .773 & 0 & .780 & .717 & .56 \\
\hline MTS (SVM) & .987 & -1.43 & .896 & -3.67 & .745 & 7.11 & -1.54 & .724 & -1.4 \\
\hline & & & & & itter & & & & \\
\hline & $\mathrm{Ex}$ & $x \operatorname{tr}$ & $\mathrm{Aq}$ & & $\mathrm{Co}$ & & Ems & $\mathrm{Op}$ & en \\
\hline Approach & $R M S E$ & $R^{2}$ & $R M S E$ & $R^{2}$ & $R M S E$ & $R^{2}$ & $R M S E \quad R^{2}$ & $R M S E$ & $R^{2}$ \\
\hline Avg & .179 & & .159 & & .175 & & .198 & .236 & \\
\hline & & nivaria & Multive & ariate $\mathrm{R}$ & gression & as using & Decision Tree & & \\
\hline $\mathrm{ST}(\mathrm{DT})$ & .173 & 6.59 & .152 & 8.61 & .165 & 11.10 & 10.80 & .214 & 17.78 \\
\hline $\operatorname{MTS}(\mathrm{DT})$ & .174 & 5.51 & .151 & 9.81 & .165 & 11.10 & 9.85 & .216 & 16.23 \\
\hline $\operatorname{MTSC}(\mathrm{DT})$ & .174 & 5.51 & .152 & 8.61 & .164 & 12.18 & 10.80 & .214 & 17.78 \\
\hline $\operatorname{ERC}(\mathrm{DT})$ & .174 & 5.51 & .152 & 8.61 & .165 & 11.10 & 10.80 & .214 & 17.78 \\
\hline $\operatorname{ERCC}(\mathrm{DT})$ & .173 & 6.59 & .153 & 7.40 & .164 & 12.18 & 10.80 & .219 & 13.89 \\
\hline MORF & .180 & -1.12 & .150 & 11.00 & .170 & 5.63 & $.180 \quad 17.36$ & .220 & 13.10 \\
\hline & & Univ: & iate $/ \mathrm{Mu}$ & ultivaria & Regres & ssions $u$ & ing SVM & & \\
\hline ST (SVM) & .181 & -2.25 & .165 & -7.69 & .183 & -9.35 & $\begin{array}{ll}.179 & 18.27\end{array}$ & .226 & 8.29 \\
\hline $\operatorname{MTS}(\mathrm{SVM})$ & .181 & -2.25 & .162 & -3.81 & .175 & 0 & 20.99 & .234 & 1.69 \\
\hline
\end{tabular}




\section{Cross-media Learning}

In this section, we explore cross-media learning by utilizing the available goldenstandard datasets to train models in different platforms when little or no training data is available.

To investigate whether we could improve predictions by expanding the training examples from one social media source to another one, we employ the three social media datasets that we explained in Section 4. An interesting difference among the three datasets is the number of labeled users, from thousands of users in Facebook, to hundreds of vloggers in YouTube, and only tens of Twitter users.

One downside in cross-media learning is that we cannot directly use the specific features related to each dataset for training the models, e.g., audio/video features extracted from vlogs or specific users' activities in Facebook. Thus, to make similar training examples, we focus on the common features that we could extract from these datasets. The common features that we use are gender and the linguistic features as we described in Section 5.1 except for the NRC features. Overall, for cross-media learning we extract 161 non zero features for each dataset.

Each dataset has used a different questionnaire for calculating the personality scores, the Facebook dataset is based on the Big Five inventory questionnaire, the YouTube dataset used Ten-Item Personality Inventory (TIPI) and personality scores of the Twitter dataset are collected using 10-item Personality Test (BFI-10). Therefore, the range of the personality scores in our Facebook dataset is between $[1,5]$, while in our YouTube dataset is between $[1,7]$ and in our Twitter dataset is between $[-0.5,0.5]$.

To obtain training examples with similar personality scores, we first map all the scores to values between $[0,1]$. For this purpose, we consider the actual score ranges of the relevant questionnaire and then map the values by using $f:[\min , \max ] \rightarrow$ $[0,1]: x \mapsto \frac{x-\min }{\max -\min }$.

Another important factor that we consider for cross-media learning regards personality dimensions. In both YouTube and Twitter datasets we have scores for Emotional Stability, however in Facebook, we have the reverse score which is Neuroticism. Thus, we convert the value in the Facebook dataset from Neuroticism (Nue) to Emotional Stability (Ems) by Ems $=1-\mathrm{Neu}$.

Figure 2 presents the distribution of the converted personality scores in all three datasets. Note that the range of the scores are between $[0,1]$, however the distributions are different which can affect the performance of the cross-media learning experiments. To evaluate the effect of cross-domain learning, we set up six experiments: (1) $\{F+Y\} \rightarrow F,(2)\{F+T\} \rightarrow F$, (3) $\{Y+F\} \rightarrow Y$, (4) $\{T+Y\} \rightarrow T$, (5) $\{T+F\} \rightarrow T$, and (6) $\{Y+T\} \rightarrow Y$, where F, Y and T stand for Facebook, YouTube and Twitter, respectively. For each of these experiments, we expand the training examples of the first dataset with the second data and apply the learned model on the testing examples of the first dataset.

In all the above experiments, we expand the training examples of one dataset with training examples of another dataset. For this task, we manually create 10 folds out of the first dataset, then each training fold is expanded with the second dataset. The results of this experiment are also averaged over 10 folds.

According to the results presented in the previous section, both $M T S C(D T)$ and $E R C C(D T)$ outperform other learners in all traits across all three datasets compared to other methods. Since the difference between the results of applying 


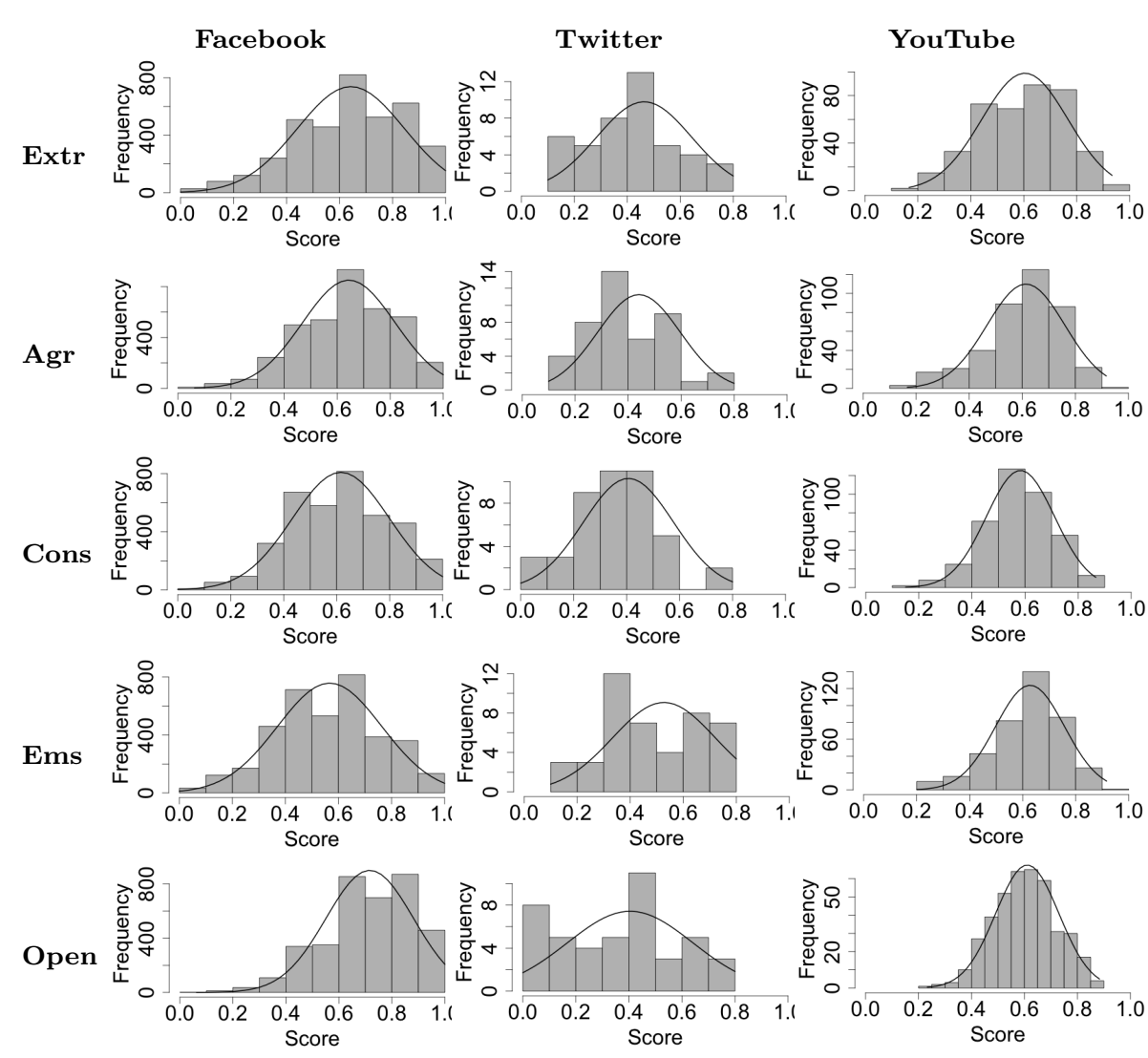

Fig. 2 Distribution of personality scores on five traits, Extroversion (Extr), Agreeableness (Agr), Conscientiousness (Cons), Emotional Stability (Ems) vs. Neuroticism (Neu), Openness (Open), in Facebook, YouTube and Twitter datasets. The black curve in each plot presents the normal distribution.

$M T S C(D T)$ and $E R C C(D T)$ on all three datasets are not significant, we choose $E R C C(D T)$ as the learning algorithm in this section. Thus, for cross-learning we only focus on the improvement achieved by expanding the training examples using $E R C C(D T)$ as a learner. To compare the results with the situation in which only training examples from the same source are used, we run $E R C C(D T)$ on the three datasets by applying common features and transformed personality scores. Thus, in addition to the above cross-learning experiments, we run three experiments (1) $F \rightarrow F,(2) Y \rightarrow Y$, and $(3) T \rightarrow T$, where F, Y and T stand for Facebook, YouTube and Twitter respectively.

Note that due to the change in size of the feature space and normalization, results of the experiments listed above are different from those presented in Table 9. The experimental results in Table 10 indicate that extending the training examples of similar datasets, namely Twitter and Facebook, is more effective than an extension with a dataset which has a different context, i.e., YouTube vlogs. It is interesting that in case of the Twitter dataset, where we only have 44 users with personality scores, extending the training examples with both Facebook and 
Table 10 Root mean square error $(R M S E)$ and Coefficient of determination $\left(R^{2}\right)$ results for the personality trait prediction using cross-media learning approaches over Facebook $(\mathrm{F})$, YouTube (Y) and Twitter (T) datasets. The five personality traits are Extroversion (Extr), Agreeableness (Agr), Conscientiousness (Cons), Emotional Stability (Ems) vs. Neuroticism (Neu), and Openness (Open). In each column, the lowest error and highest determination are typeset in bold. Significant differences with respect to the baseline $(p<.05)$ are marked using *. Baseline is the average baseline which is shown by $A v g$.

\begin{tabular}{|c|c|c|c|c|c|c|c|c|c|c|}
\hline \multirow[b]{2}{*}{ Approach } & \multicolumn{2}{|c|}{ Extr } & \multicolumn{2}{|c|}{ Agr } & \multicolumn{2}{|c|}{ Cons } & \multicolumn{2}{|c|}{ Ems } & \multicolumn{2}{|c|}{ Open } \\
\hline & $R M S E$ & $R^{2}$ & $R M S E$ & $R^{2}$ & $R M S E$ & $R^{2}$ & $R M S E$ & $R^{2}$ & $R M S E$ & $R^{2}$ \\
\hline \multicolumn{11}{|c|}{ Facebook } \\
\hline Avg & .202 & & .175 & & .184 & & .196 & & .165 & \\
\hline $\mathrm{F} \rightarrow \mathrm{F}$ & .199 & 2.94 & .173 & 2.27 & .179 & 5.36 & .192 & 4.04 & .163 & 2.41 \\
\hline$\{\mathrm{F}+\mathrm{Y}\} \rightarrow \mathrm{F}$ & .199 & 2.94 & .173 & 2.27 & .180 & 4.30 & .192 & 4.04 & .162 & 3.60 \\
\hline$\{\mathrm{F}+\mathrm{T}\} \rightarrow \mathrm{F}$ & .199 & 2.94 & .173 & 2.27 & .180 & 4.30 & .192 & 4.04 & .163 & 2.41 \\
\hline \multicolumn{11}{|c|}{ YouTube } \\
\hline Avg & .163 & & .147 & & .129 & & .130 & & .120 & \\
\hline $\mathrm{Y} \rightarrow \mathrm{Y}$ & .154 & 10.73 & $.121^{*}$ & 32.25 & .116 & 19.14 & $.117^{*}$ & $19^{*}$ & .118 & 3.31 \\
\hline$\{\mathrm{Y}+\mathrm{F}\} \rightarrow \mathrm{Y}$ & .153 & 11.89 & .130 & 21.79 & .117 & 17.74 & .121 & 13.37 & $.116^{*}$ & $6.55^{*}$ \\
\hline$\{\mathrm{Y}+\mathrm{T}\} \rightarrow \mathrm{Y}$ & .153 & 11.89 & .123 & 29.99 & $.115^{*}$ & $20.53^{*}$ & .119 & 16.21 & $.116^{*}$ & $6.55^{*}$ \\
\hline \multicolumn{11}{|c|}{ Twitter } \\
\hline Avg & .179 & & .159 & & .175 & & .198 & & .236 & \\
\hline $\mathrm{T} \rightarrow \mathrm{T}$ & $.171^{*}$ & $8.74^{*}$ & .151 & 9.81 & .166 & 10.02 & .183 & 14.58 & .216 & 16.23 \\
\hline$\{\mathrm{T}+\mathrm{F}\} \rightarrow \mathrm{T}$ & $.170^{*}$ & $9.80^{*}$ & .156 & 3.74 & .165 & 11.10 & .186 & 11.75 & .226 & 9.96 \\
\hline$\{\mathrm{T}+\mathrm{Y}\} \rightarrow \mathrm{T}$ & $.175^{*}$ & $4.42^{*}$ & .161 & -9.93 & .177 & -2.30 & .184 & 13.64 & .222 & 11.51 \\
\hline
\end{tabular}

YouTube examples indicates no improvement over the training examples of the same source. Besides the Twitter dataset, for the case of YouTube and Facebook, we also gain little or no improvement using the training examples of other sources. These results indicate that the context and respectively the users of these social media sites are different, which is in line with the distribution of the personality scores in Figure 2, Besides the context, the way that the personality scores were calculated are different among these datasets (i.e., observed vs. self-reported), which may also influence the performance of the cross-media learners. Furthermore, having more training examples of the same source makes the performance of the learner more stable, therefore for the case of the Facebook dataset, the performance of the learner by extending the examples with both the YouTube and the Twitter datasets do not differ much. These results differ from the results of cross-media learning of Farnadi et al. in [16, where the performance of the learner was improved by an extension of the training examples. These results suggest that the success of cross-media learning is very dependent on the similarity of two data sources w.r.t. the distribution and calculation of the personality scores.

\section{Discussion, Conclusion and Future Directions}

In this study, we performed a comparative analysis of state-of-the-art computational personality recognition methods on a varied set of social media ground truth data from Facebook, Twitter and YouTube. We attempted to address three research questions as follows.

(1) Should personality prediction be treated as a multi-label prediction task (i.e., all personality traits of a given user are predicted at once), or 
should each trait be identified separately? We leveraged a variety of univariate (i.e., decision tree and support vector machine) and multivariate regression techniques (i.e., multi-target stacking, ensemble of regressor chains, and multiobjective random forests) as presented in Section 5.2. When using these learners on the three different datasets, decision tree models mostly outperformed support vector machine models, while multivariate regression learners with decision tree as a base learner often outperformed the univariate regression ones. The differences between univariate and multivariate models were not significant though. Overall the best performing models for this task are the Multi-Target Stacking Corrected $(M T S C)$ model and the Ensemble of Regressor Chains Corrected (ERCC) model by using a decision tree as a base learner.

(2) Which predictive features work well across different on-line environments? To address this question, we utilized different content-based features (e.g., linguistic features such as LIWC) and context-based features (e.g., audio and video features extracted from vlog videos) in each dataset. We analyzed the correlation between features and personality traits in Section 6.1. We collected the common correlated features with traits among three datasets. From 166 common features for five traits, only 15 common correlations were found. These results suggested that it may not be possible to generalize the correlation between features and the personality traits, as it may vary depending on the underlying data.

Moreover, we measured the performance of the models using different feature sets in addition to the corresponding correlated subset of features. For the YouTube and Facebook datasets, among different feature sets, the LIWC feature set outperformed others for predicting the personality scores of all traits. From the results using both the original feature set and the corresponding correlated feature set, we concluded that selecting features and only using correlated features does not necessarily increase the performance of the learner, however by reducing the size of the feature space we are able to increase the efficiency of the algorithm. Due to the large number of social media users, there is a need to explore efficient models with high performance. Thus, exploring the smallest feature set without loosing the performance in predicting personality traits is an interesting future direction. Furthermore, in this study, we considered Pearson and Spearman correlation as a feature selection approach, however investigating other measures for computing the correlations between features and the personality trait such as information gain [34] is an open path to explore.

(3) What is the decay in accuracy when porting models trained in one social media environment to another? To answer this question, we conducted six cross-media learning experiments in which we expand the training examples of one dataset using another dataset. The results were presented in Section 7 .

Expanding a model with training examples from another source has not improved the performance of the learner. The context of the data plays a major role in the success of cross-media learning. Since our YouTube dataset was labeled as perceived personality scores compared to self-reported ones in the case of Facebook and Twitter, a complementary study on the effects of using similar data sources w.r.t. the variation among users and the method for collecting the personality scores in cross-media learning remains a topic for future work. 


\section{Acknowledgements}

We would like to thank the anonymous reviewers for their helpful comments and suggestions. This work was funded in part by the SBO-program of the Flemish Agency for Innovation by Science and Technology (IWT-SBO-Nr. 110067).

\section{References}

1. Aharony, N., Pan, W., Ip, C., Khayal, I., Pentland, A.: Social fmri: Investigating and shaping social mechanisms in the real world. Pervasive and Mobile Computing 7(6), 643659 (2011)

2. Aran, O., Gatica-Perez, D.: Cross-domain personality prediction: from video blogs to small group meetings. In: Proceedings of the 15th ACM International conference on multimodal interaction, pp. 127-130. ACM (2013)

3. Bachrach, Y., Kosinski, M., Graepel, T., Kohli, P., Stillwell, D.: Personality and patterns of Facebook usage. In: Proceedings of the 3rd Annual ACM Web Science Conference (Web-Sci), pp. 24-32. ACM (2012)

4. Back, M.D., Stopfer, J.M., Vazire, S., Gaddis, S., Schmukle, S.C., Egloff, B., Gosling, S.D.: Facebook profiles reflect actual personality, not self-idealization. Psychological Science 21, 372-374 (2010)

5. Bai, S., Hao, B., Li, A., Yuan, S., Gao, R., Zhu, T.: Predicting Big Five personality traits of microblog users. In: Proceedings of the IEEE/WIC/ACM WI-IAT, vol. 1, pp. 501-508 (2013)

6. Biel, J., Gatica-Perez, D.: The YouTube lens: Crowdsourced personality impressions and audiovisual analysis of vlogs. Multimedia, IEEE Transactions on 15(1), 41-55 (2013)

7. Biel, J.I., Aran, O., Gatica-Perez, D.: You are known by how you vlog: Personality impressions and nonverbal behavior in youtube. In: Proceedings of the AAAI International Conference on Weblogs and Social Media (ICWSM), pp. 446-449 (2011)

8. Blockeel, H., Raedt, L.D., Ramon, J.: Top-down induction of clustering trees. In: Proceedings of the Fifteenth International Conference on Machine Learning, pp. 55-63 (1998)

9. Cantador, I., Fernández-Tobías, I., Bellogín, A., Kosinski, M., Stillwell, D.: Relating personality types with user preferences in multiple entertainment domains. In: Proceedings of the 1st workshop on emotion and personality in personalized services (EMPIRE) (2013)

10. Celli, F., Lepri, B., Biel, J.I., Gatica-Perez, D., Riccardi, G., Pianesi, F.: The workshop on computational personality recognition 2014. In: Proceedings of the ACM International Conference on Multimedia, pp. 1245-1246. ACM (2014)

11. Celli, F., Rossi, L.: The role of emotional stability in Twitter conversations. In: Proceedings of the Workshop on Semantic Analysis in Social Media. Association for Computational Linguistics, pp. 10-17 (2012)

12. Costa, P.T., McCrae, R.R.: The Revised NEO Personality Inventory (NEO-PI-R). The SAGE Handbook Of Personality Theory And Assessment 2, 179-198 (2008)

13. Counts, S., Stecher, K.: Self-presentation of personality during online profile creation. In: Proceedings of the International AAAI Conference on Weblogs and Social Media (ICWSM), pp. 191-194 (2009)

14. Farnadi, G., Sitaraman, G., Rohani, M., Kosinski, M., Stillwell, D., Moens, M., Davalos, S., De Cock, M.: How are you doing? Emotions and personality in Facebook. In: Proceedings of the EMPIRE, pp. 45-56 (2014)

15. Farnadi, G., Sushmita, S., Sitaraman, G., Ton, N., De Cock, M., Davalos, S.: A Multivariate Regression Approach to Personality Impression Recognition of Vloggers. In: Proceedings of the WCPR, pp. 1-6 (2014)

16. Farnadi, G., Zoghbi, S., Moens, M., De Cock, M.: Recognising personality traits using Facebook status updates. In: Proceedings of the WCPR, pp. 14-18 (2013)

17. Gill, A.J., Oberlander, J., Austin, E.: Rating e-mail personality at zero acquaintance. Personality and Individual Differences 40(3), 497-507 (2006)

18. Giota, K.G., Kleftaras, G.: The role of personality and depression in problematic use of social networking sites in greece. Journal of Psychosocial Research on Cyberspace $7(3)$ (2013) 
19. Golbeck, J., Robles, C., Edmondson, M., Turner, K.: Predicting personality from twitter. In: Privacy, Security, Risk and Trust (PASSAT) and 2011 IEEE Third Inernational Conference on Social Computing (SocialCom), 2011 IEEE Third International Conference on, pp. 149-156. IEEE (2011)

20. Golbeck, J., Robles, C., Turner, K.: Predicting personality with social media. In: CHI'11 Extended Abstracts on Human Factors in Computing Systems, pp. 253-262. ACM (2011)

21. Goldberg, L.R., Johnson, J.A., Eber, H.W., Hogan, R., Ashton, M.C., Cloninger, C.R., Gough, H.G.: The international personality item pool and the future of public-domain personality measures. Journal of Research in Personality 40(1), 84-96 (2006)

22. Guyon, I., Elisseeff, A.: An introduction to variable and feature selection. The Journal of Machine Learning Research 3, 1157-1182 (2003)

23. Hagger-Johnson, G., Egan, V., Stillwell, D.: Are social networking profiles reliable indicators of sensational interests? Journal of Research in Personality 45(1), 71 - 76 (2011)

24. Hall, M.A.: Correlation-based feature selection for machine learning. Ph.D. thesis, The University of Waikato (1999)

25. $\mathrm{Hu}, \mathrm{R} ., \mathrm{Pu}, \mathrm{P} .:$ Enhancing collaborative filtering systems with personality information. In: Proceedings of the ACM RecSys, pp. 197-204 (2011)

26. Hughes, D.J., Rowe, M., Batey, M., Lee, A.: A tale of two sites: Twitter vs. Facebook and the personality predictors of social media usage. Computers in Human Behavior 28(2), 561-569 (2012)

27. Iacobelli, F., Culotta, A.: Too Neurotic, Not Too Friendly: Structured Personality Classification on Textual Data. In: Proceedings of the Workshop on Computational Personality Recognition, AAAI Press, Melon Park, CA, pp. 19-22 (2013)

28. John, O.P., Srivastava, S.: The Big Five trait taxonomy: History, measurement, and theoretical perspectives. Handbook of Personality: Theory and Research 2, 102-138 (1999)

29. Jolliffe, I.: Principal component analysis. Wiley Online Library (2002)

30. Kocev, D., Vens, C., Struyf, J., Džeroski, S.: Ensembles of multi-objective decision trees. In: Proceedings of the ECML, pp. 624-631 (2007)

31. Kosinski, M., Bachrach, Y., Kohli, P., Stillwell, D., Graepel, T.: Manifestations of user personality in website choice and behaviour on online social networks. Machine Learning pp. 1-24 (2013)

32. Kosinski, M., Stillwell, D.J., Graepel, T.: Private traits and attributes are predictable from digital records of human behavior. Proceedings of the National Academy Of Sciences (PNAS) 110, 5802-5805 (2013)

33. Lambiotte, R., Kosinski, M.: Tracking the Digital Footprints of Personality. Proceedings of the Institute of Electrical and Electronics Engineers (PIEEE) pp. 1935-1939 (2014)

34. Lee, C., Lee, G.G.: Information gain and divergence-based feature selection for machine learning-based text categorization. Information Processing \& Management 42(1), 155-165 (2006)

35. Lee, K.M., Nass, C.: Designing social presence of social actors in human computer interaction. In: Proceedings of the SIGCHI Conference on Human Factors in Computing Systems, CHI '03, pp. 289-296. ACM (2003)

36. Mairesse, F., Walker, M.A., Mehl, M.R., Moore, R.K.: Using linguistic cues for the automatic recognition of personality in conversation and text. Journal of Artificial Intelligence Research 30, 457-501 (2007)

37. Mohammad, S., Zhu, X., Martin, J.: Semantic role labeling of emotions in tweets. In: Proceedings of the WASSA, pp. 32-41 (2014)

38. Mohammad, S.M., Kiritchenko, S.: Using nuances of emotion to identify personality. arXiv preprint arXiv:1309.6352 (2013)

39. Nguyen, T., Phung, D.Q., Adams, B., Venkatesh, S.: Towards discovery of influence and personality traits through social link prediction. In: Proceedings of ICWSM, pp. 566-569 (2011)

40. de Oliveira, R., Karatzoglou, A., Cerezo, P.C., de Vicuña, A.A.L., Oliver, N.: Towards a psychographic user model from mobile phone usage. In: Proceedings of the International Conference on Human Factors in Computing Systems, CHI, pp. 2191-2196 (2011)

41. Oliveira, R.D., Cherubini, M., Oliver, N.: Influence of personality on satisfaction with mobile phone services. ACM Transactions on Computer Human Interaction 20(2), 10:110:23 (2013)

42. Ozer, D.J., Benet-Martinez, V.: Personality and The Prediction of Consequential Outcomes. Annual Review of Psychology 57, 401-421 (2006) 
43. Park, G., Schwartz, H.A., Eichstaedt, J.C., Kern, M.L., Kosinski, M., Stillwell, D.J., Ungar, L.H., Seligman, M.E.: Automatic personality assessment through social media language. Journal of personality and social psychology 108(6), 934 (2015)

44. Pennebaker, J.W., King, L.A.: Linguistic styles: language use as an individual difference. Journal of personality and social psychology $\mathbf{7 7}(6), 1296$ (1999)

45. Polzehl, T., Moller, S., Metze, F.: Automatically assessing personality from speech. In: Semantic Computing (ICSC), 2010 IEEE Fourth International Conference on, pp. 134-140. IEEE (2010)

46. Quercia, D., Kosinski, M., Stillwell, D., Crowcroft, J.: Our Twitter profiles, our selves: Predicting personality with Twitter. In: Privacy, Security, Risk and Trust (passat), 2011 IEEE Third International Conference on Social Computing (socialcom), pp. 180-185. IEEE (2011)

47. Quercia, D., Lambiotte, R., Kosinski, M., Stillwell, D.J., Crowcroft, J.: The personality of popular Facebook users. In: Proceedings of the Conference on Computer Supported Cooperative Work, pp. 955-964 (2012)

48. R Core Team: R: A Language and Environment for Statistical Computing. R Foundation for Statistical Computing, Vienna, Austria (2014). URL http://www.R-project.org

49. Rammstedt, B., John, O.P.: Measuring personality in one minute or less: A 10-item short version of the Big Five Inventory in English and German. Journal of research in Personality 41(1), 203-212 (2007)

50. Saati, B., Salem, M., Brinkman, W.P.: Towards customized user interface skins: investigating user personality and skin colour. Proceedings of the HCI 2005 2, 89-93 (2005)

51. Schwartz, H.A., Eichstaedt, J.C., Kern, M.L., Dziurzynski, L., Ramones, S.M., Agrawal, M., Shah, A., Kosinski, M., Stillwell, D., Seligman, M.E., et al.: Personality, gender, and age in the language of social media: The open-vocabulary approach. PloS one 8(9), e73,791 (2013)

52. Stillwell, D.J., Kosinski, M.: myPersonality Project Website. myPersonality Project (2015). URL http://mypersonality.org

53. Tausczik, Y.R., Pennebaker, J.W.: The Psychological meaning of words: LIWC and computerized text analysis methods. Journal of Language and Social Psychology 29, 24-54 (2010)

54. Xioufis, E.S., Groves, W., Tsoumakas, G., Vlahavas, I.P.: Multi-label classification methods for multi-target regression. arXiv preprint arXiv:1211.6581 (2012)

55. Youyou, W., Kosinski, M., Stillwell, D.J.: Computer-based personality judgements are more accurate than those made by humans. Proceedings of The National Academy of Sciences (PNAS) 112(4), 1036-1040 (2015) 
Vitae

1. Golnoosh Farnadi

Department of Applied Mathematics, Computer Science and Statistics, Ghent University, Krijgslaan 281, B-9000 Ghent, Belgium

Department of Computer Science, Katholieke Universiteit Leuven, Celestijnenlaan 200A, B-3001 Heverlee, Belgium

Golnoosh Farnadi is a joint $\mathrm{PhD}$ student in Computer science from Ghent University and Katholieke Universiteit Leuven. She received her M.Sc. and B.Sc in Computer science from Delft university of Technology in 2011 and Shahid Beheshti university in 2007 respectively. She was a visiting scholar in the Center for Data Science at the University of Washington Tacoma and a visiting scholar in the Statistical Relational Learning Group at the University of California Santa Cruz. Her current research interests are machine learning, statistical relational learning, artificial intelligence, social networks, user modeling and big data.

\section{Geetha Sitaraman}

Center for Data Science, University of Washington Tacoma, 1900 Commerce St, Tacoma, WA USA 98402

Geetha Sitaraman received her M.S. in Computer Science from the University of Washington, Tacoma in 2014, and B.S. in Electrical and Electronic Engineering from University of Madras, India in 2003. She has worked primarily in the area of user modeling, specifically on applying different machine learning techniques and feature selection methodologies, for inferring latent user attributes, like personality and emotions, on online social networks. Her primary interests lie in the areas of studying personalized systems, like personality and/or emotion-aware interfaces, which are more persuasive.

\section{Shanu Sushmita}

Center for Data Science, University of Washington Tacoma, 1900 Commerce St, Tacoma, WA USA 98402

Shanu Sushmita is a Postdoctoral Research Scientist at Center for Data Science, UW Tacoma. Her research interests are in the area of Information Retrieval, Machine Learning, and Healthcare analytics. She received her $\mathrm{PhD}$ from the University of Glasgow in 2012, and Bachelors in Engineering from the North Maharashtra University 2004. Her current research is focused on providing machine-learning solutions in healthcare (e.g., improving online health information search, healthcare cost prediction, predicting risk of hospital readmission, etc).

\section{Fabio Celli}

Center for Mind/Brain Sciences, University of Trento, Palazzo Fedrigotti corso Bettini 31, 38122 Trento, Italy 
Fabio Celli, $\mathrm{PhD}$, is a research fellow at the department of computer science at the University of Trento, Italy. He has a background in Communication Studies and Computational Linguistics, his interest are related to computational recognition of personality, brand personality, natural language processing and data mining. He is in the organizing commitee of the Workshop on Computational Persoanlity Recognition (WCPR) and the workshop on author profiling (PAN). He has been a reviewer for numerous conferences and journals, including Computational Linguistics (journal), Scientific Reports (journal), Computational Intelligence (journal) and Socinfo (conference).

\section{Michal Kosinski}

Stanford Graduate School of Business, Stanford University, 665 Knight Way, Stanford, CA 94305

Michal Kosinski is the Assistant Professor in Organizational Behavior at the Graduate School of Business, Stanford University. After receiving his $\mathrm{PhD}$ in Psychology from the University of Cambridge (UK) in 2014, Kosinski spent a year as a Postdoctoral Scholar at the Computer Science Department at Stanford University. Kosinskis research had a significant impact on both academia and the industry. His findings featured in The Economists special report on the future of insurance (2015), inspired two TED talks, and prompted a discussion in the EU Parliament. In 2013, Kosinski was listed among the 50 most influential people in Big Data by DataIQ and IBM, while two of his papers were placed in the Top 100 Papers That Most Caught the Public Imagination (\#9 and \#21).

\section{David Stillwell}

Judge Business School, University of Cambridge, CB2 1AG, UK

David Stillwell is a lecturer in Big Data Analytics and Computational Social Science at Cambridge University's Judge Business School. He received his B.Sc., M.Sc. and PhD in Psychology from the University of Nottingham in 2007, 2008 and 2012 respectively. His research takes advantage of large datasets of 'digital footprints,' which are generated when individuals interact via digital services such as social networks or credit cards. This data is used to understand behaviour in the real world.

\section{Sergio Davalos}

Milgard School of Business, University of Washington Tacoma, 1900 Commerce St, Tacoma, WA, USA 98402

Dr. Sergio Davalos is Associate Professor of MIS at University of Washington Tacoma. He is a member of the Center for Information Based Management and the Center for Data Science at the University of Washington Tacoma. Dr. Davalos received his B.A. degree in Mathematics from the University of Arizona, his M.S. in Computer Science from the University of Arizona University, and his and Ph.D. degree in Management Information Systems from the University of Arizona University. Dr. Davalos has worked in several areas of artificial intelligence and machine learning including expert systems, knowledge 
representation, neural networks, ant colony optimization, and data mining. He has authored papers ranging from applications of machine learning in psychology, finance, accounting, and logistics and design science for human computer interaction. His most recent papers involve text mining of user generated content on social platforms as well as text mining of government repositories.

\section{Marie-Francine Moens}

Department of Computer Science, Katholieke Universiteit Leuven, Celestijnenlaan 200A, B-3001 Heverlee, Belgium

Marie-Francine (Sien) Moens is a full professor at the Department of Computer Science of the Katholieke Universiteit Leuven, Belgium. She holds a M.Sc. and a Ph.D. degree in Computer Science from this university. She is head of the Language Intelligence and Information retrieval (LIIR) research group, and is a member of the Human Computer Interaction unit. She is currently also head of the Informatics section of the Department of Computer Science at KU Leuven. She is author or co-author of more than 290 international, peer-reviewed publications. Her main interests are in the domain of automated content recognition in text and multimedia data and its application in information extraction and retrieval using statistical machine learning, and exploiting insights from linguistic and cognitive theories.

\section{Martine De Cock}

Center for Data Science, University of Washington Tacoma, 1900 Commerce St, Tacoma, WA USA 98402

Dr. Martine De Cock holds a M.Sc. and a Ph.D. degree in Computer Science from Ghent University. She is an associate professor at the Institute of Technology, University of Washington Tacoma, as well as a guest professor at Ghent University. Her previous work experiences include positions as a research assistant and a postdoctoral fellow supported by the Fund for Scientific Research - Flanders, a visiting scholar in the BISC group at the University of California, Berkeley, a visiting scholar at the Knowledge Systems Laboratory at Stanford University, and an associate professor at the Department of Applied Mathematics, Computer Science and Statistics at Ghent University. She has over 150 peer reviewed publications in international journals and conferences on artificial intelligence, data mining, machine learning, information retrieval, web intelligence and logic programming. She is a program committee member of numerous international conferences and an associate editor of IEEE Transactions on Fuzzy Systems. She co-organized the KDDCup2013. Her current research interests are machine learning on big data, social networks, and big data analytics to improve the quality of healthcare. 